\title{
A modified side-sensitive synthetic chart to monitor the process mean
}

\author{
S.C. Shongwe* and M.A. Graham \\ Department of Statistics \\ University of Pretoria \\ South Africa
}

\begin{abstract}
In this paper, we propose a modified side-sensitive (MSS) synthetic $\bar{X}$ chart which signals only if all the consecutive plotting statistics that lead to an out-of-control event fall on one side of the center line; unlike the non-side-sensitive, standard and revised side-sensitive synthetic $\bar{X}$ charts that also signal even when some of the plotting statistics fall on opposite sides of the center line. Moreover, we use the Markov chain imbedding technique to study and compare the zero-state and steady-state average run-length $(A R L)$, extra quadratic loss, average ratio of the $A R L s$ and performance comparison index of the proposed MSS chart with other Shewhart-type synthetic and runs-rules charts. The synthetic $\bar{X}$ chart with this MSS feature has a better overall zero-state and steady-state performance than the existing synthetic $\bar{X}$ charts and hence makes it a strong contender in many applications where existing synthetic $\bar{X}$ charts are currently used.
\end{abstract}

Keywords: Runs-rules, Side-sensitive, Steady-state, Synthetic chart, Zero-state

\section{Introduction}

Statistical process control and monitoring (SPCM) is a collection of statistical techniques and tools which allows high quality products to be produced. Among the SPCM tools, control charts are undeniably the most widely used for identifying changes in processes. Control charts are mainly used to distinguish between the chance and the assignable causes of variation. When a process has only chance causes of variation present, it is said to be statistically in-control (IC), otherwise, the process is said to be out-of-control (OOC).

Assume that $\left\{X_{i j}: i \geq 1 ; j=1,2, \ldots, n\right\}$ is a sequence of samples from iid $N\left(\mu_{0}, \sigma_{0}^{2}\right)$ distribution where $\mu_{0}$ and $\sigma_{0}^{2}$ are the specified IC mean and variance, respectively. Let $\bar{X}_{i}$ denote the

\footnotetext{
* Corresponding author: SC Shongwe (sandile.shongwe@up.ac.za)
} 
plotting statistic calculated from $\left\{X_{i j}\right\}$ at sampling point $i$. A control chart that is usually used to monitor $\bar{X}_{i}$ is called the $\bar{X}$ chart and it signals when a single plotting statistic falls above the upper control limit $(U C L)$ or below the lower control limit $(L C L)$ which are given by

$$
U C L=\mu_{0}+k \sigma_{0}, C L=\mu_{0}, L C L=\mu_{0}-k \sigma_{0},
$$

where $k$ is the distance of the control limits from the center line $(C L)$; see Figure 1. A Shewhart $\bar{X}$ chart is known to be more efficient in detecting large process shifts, however, it is relatively insensitive in detecting small and moderate shifts. In order to increase the sensitivity of the $\bar{X}$ chart for detecting smaller shifts, Wu and Spedding [24] proposed a synthetic $\bar{X}$ chart for the mean which consists of two sub-charts, one, a basic $\bar{X}$ chart and a second, a conforming run-length $(C R L)$ chart. For a synthetic chart, an OOC signal is not based on a single plotting statistic falling beyond the control limits in Equation (1), instead, when a sample produces a value beyond the control limits in Equation (1), that sample is marked as nonconforming and the control procedure moves to the second sub-chart and a signal is obtained depending on the outcome of the $C R L$ sub-chart. Note that when a sample produces a value falling between $L C L$ and $U C L$, then that sample is marked as conforming. A CRL is defined as the number of conforming samples between two consecutive nonconforming samples, inclusive of the nonconforming sample at the end; see Bourke [6]. Note that the absence of any conforming samples between two nonconforming samples leads to the minimum $C R L$ i.e. $C R L=1$. The $C R L$ chart signals when an observed $C R L$ value is less than or equal to some threshold, say $H$ (an integer, greater or equal to 1), which is defined to be the threshold / control limit of the $C R L$ chart. Note though a more general definition of synthetic charts is given by Scariano and Calzada [22], where they stated that a synthetic chart "consists of any control charting procedure operating in union with the $C R L$ chart". To make the run-length analysis of the synthetic chart easier, Davis and Woodall [10] showed that a synthetic chart is a special case of a runs-rules chart i.e. a 2-of-(H+1) with a head-start (HS) feature. The HS feature implies that we assume that (at time 0) the first observation is nonconforming, consequently, we need at least one 
other nonconforming sample within the next $H$ sampling points, for a $2-o f-(H+1)$ runs-type chart to issue a signal.

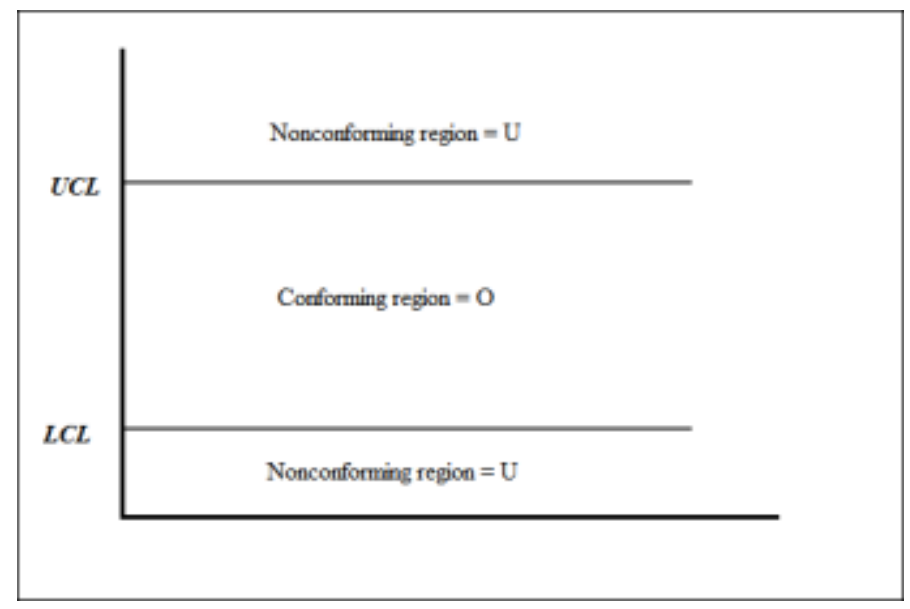

(a) Non-side-sensitive regions

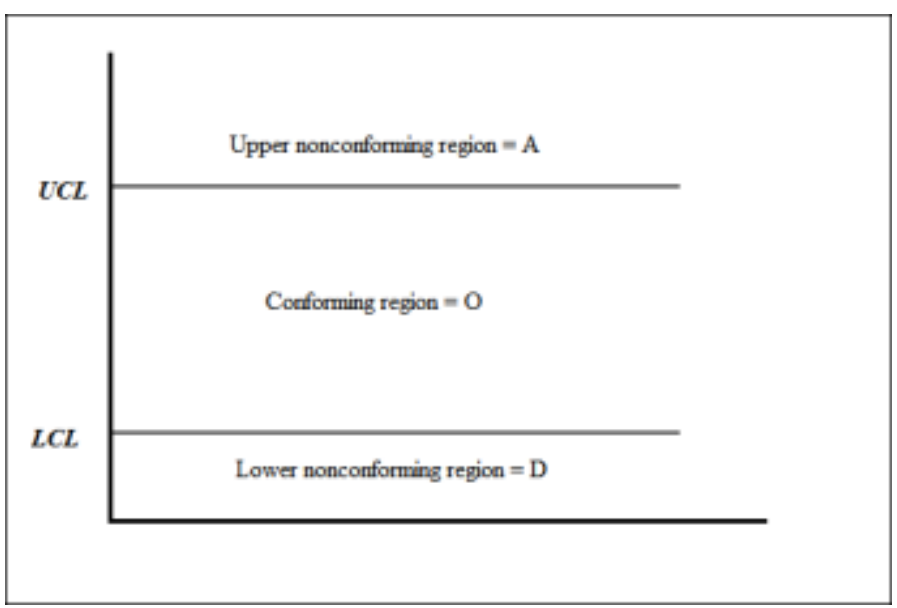

(b) Standard / revised side-sensitive regions

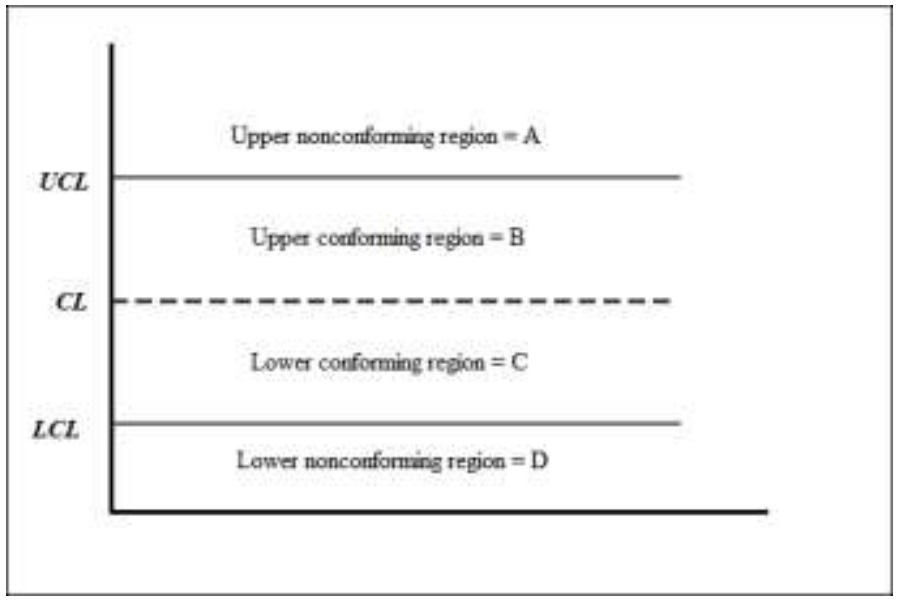

(c) Modified side-sensitive regions

Figure 1: The control limits and the corresponding regions of the $\bar{X}$ sub-chart 
We can classify the available synthetic charts that are based on the sub-chart limits in Equation (1) into three types which are as follows:

(i) the non-side-sensitive synthetic chart (by Wu and Spedding [24] - denoted by WS) that signals when two nonconforming samples out of $H+1$ successive samples fall outside the control limits, irrespective of whether one (or both) of the nonconforming samples fall above the $U C L$ and the other (or both) falls below the $L C L$, which are separated by at most $H$-1 conforming samples that fall between the $L C L$ and the $U C L$. The control charting regions are shown in Figure 1(a).

(ii) the standard side-sensitive synthetic chart (by Davis and Woodall [10] - denoted by DW) that signals when two nonconforming samples out of $H+1$ successive samples fall above (below) the $U C L(L C L)$ which are separated by at most $H-1$ samples that fall below (above) the $U C L(L C L)$, respectively. The control charting regions are shown in Figure 1(b).

(iii) the revised side-sensitive synthetic chart (by Machado and Costa [18] - denoted by MC) that signals when two nonconforming samples out of $H+1$ successive samples fall above (below) the $U C L(L C L)$ which are separated by at most $H-1$ conforming samples that fall between the $L C L$ and the $U C L$, respectively. The control charting regions are shown in Figure 1(b).

Before discussing the aim of this paper, we also differentiate between the different types of runsrules charts (without the HS feature). For the control limits in Equation (1), there are four types of runs-rules that are considered in the literature and these are as follows (where $w \geq 2$ and $v \geq 0$ are specified positive integers):

(i) the non-side-sensitive $w$-of- $(w+v)$ (by Derman and Ross [11] - denoted by DR) that signals when $w$ nonconforming samples out of $w+v$ successive samples fall outside the control limits, no matter whether some (or all) of the $w$ nonconforming samples fall above the $U C L$ and the others (or all) fall below the $L C L$, which are separated by at most $v$ conforming 
samples that fall between the $L C L$ and the $U C L$. The control charting regions are shown in Figure 1(a).

(ii) the standard side-sensitive $w$-of- $(w+v)$ (by Klein [16] - denoted by KL) that signals when $w$ nonconforming samples out of $w+v$ successive samples fall above (below) the $U C L(L C L)$ which are separated by at most $v$ samples that fall below (above) the $U C L(L C L)$, respectively. The control charting regions are shown in Figure 1(b).

(iii) the revised side-sensitive $w$-of- $(w+v)$ (adopted from the synthetic chart of Machado and Costa [18]) that signals when $w$ nonconforming samples out of $w+v$ successive samples fall above (below) the $U C L(L C L)$ which are separated by at most $v$ conforming samples that fall between the $L C L$ and the $U C L$, respectively. The control charting regions are shown in Figure 1(b).

(iv) the modified side-sensitive (MSS) $w$-of- $(w+v)$ (by Antzoulakos and Rakitzis [5] - denoted by AR) that signals when $w$ nonconforming samples out of $w+v$ successive samples fall above (below) the $U C L(L C L)$ which are separated by at most $v$ conforming samples that are plotted between the $C L$ and the $U C L(L C L)$, respectively. The control charting regions are shown in Figure 1(c).

Note that unlike the signaling rules proposed by Klein [16] and Machado and Costa [18], the signaling rules proposed by Antzoulakos and Rakitzis [5] signifies the importance of a $C L$ because the AR scheme signals when all the $w+v$ consecutive samples that lead to an OOC event are on one side of the $C L$.

Table 1: Types of runs-rules and synthetic $\bar{X}$ charts

\begin{tabular}{|c|c|}
\hline Runs-rules charts & Synthetic charts \\
\hline $\begin{array}{l}\text { (i) Non-side-sensitive } w \text {-of- }(w+v) \text { : (DR) } \\
\text { - Derman and Ross [11] }\end{array}$ & $\begin{array}{l}\text { (i) Non-side-sensitive 2-of- }(H+1) \text { : (WS) } \\
\bullet \quad \mathrm{Wu} \text { and Spedding [24] }\end{array}$ \\
\hline $\begin{array}{l}\text { (ii) Standard side-sensitive } w \text {-of- }(w+v) \text { : (KL) } \\
\text { - Klein [16] }\end{array}$ & $\begin{array}{l}\text { (ii) Standard side-sensitive 2-of- }(H+1) \text { : (DW) } \\
\text { - Davis and Woodall [10] }\end{array}$ \\
\hline $\begin{array}{l}\text { (iii) Revised side-sensitive } w \text {-of- }(w+v) \text { : (MC1) } \\
\text { - Adopted from Machado and Costa [18] }\end{array}$ & $\begin{array}{l}\text { (iii) Revised side-sensitive 2-of- }(H+1) \text { : (MC2) } \\
\text { - Machado and Costa [18] }\end{array}$ \\
\hline $\begin{array}{l}\text { (iv) Modified side-sensitive } w \text {-of- }(w+v) \text { : (AR) } \\
\text { - Antzoulakos and Rakitzis [5] }\end{array}$ & $\begin{array}{l}\text { (iv) Modified side-sensitive 2-of- }(H+1) \text { : (MSS) } \\
\text { - Proposed in this paper }\end{array}$ \\
\hline
\end{tabular}


The abovementioned four runs-rules charts and three synthetic charts are summarized in Table 1. For a fair comparison with synthetic charts, we only consider runs-rules charts with $w=2$ so that $v=H-1$ and $w+v=H+1$. To differentiate between the runs-rules and synthetic schemes by Machado and Costa [18], we denote these as MC1 and MC2, respectively. The three types of synthetic charts in Table 1 (i.e. WS, DW and MC2) have runs-type rules similar to those in DR, KL and MC1, respectively. Therefore the aim of this paper is to supplement on the work done by the authors listed in Table 1, by proposing a synthetic $\bar{X}$ chart that has runs-type rules similar to those in Antzoulakos and Rakitzis [5], called the MSS synthetic $\bar{X}$ chart. That is, this paper makes a contribution to both synthetic and runs-rules charts by:

- $\quad$ proposing a new Shewhart-type synthetic $\bar{X}$ chart;

- using a Markov chain imbedding technique to study the run-length properties of the synthetic charts and we use a design criterion with more emphasis on the overall performance to study the effectiveness of the 2-of- $(H+1)$ runs-type charts as $H$ increases i.e. $H \leq 20$. Note that most studies on runs-rules usually concentrate on $H \leq 3$;

- we give recommendations on what the optimal value of $H$ should be so that the corresponding 2-of-(H+1) runs-type chart (i.e. MSS, MC2, DW, WS, AR, MC1, KL, DR) each results in the best overall performance; depending on the upper bound on the range of shifts (later denoted by $\delta_{\max }$ ).

- To supplement on the zero-state average run-length $(A R L)$ performance of the 2-of- $(H+1)$ $\mathrm{DR}, \mathrm{KL}$ and AR, we study the steady-state $A R L$ performance of these charts. Moreover, we evaluate the zero-state performance of MC2 scheme proposed in Machado and Costa [18] and then propose its zero-state and steady-state runs-rules version i.e. MC1.

The goal of this paper is to compare a variety of simple Shewhart synthetic and runs-rules $\bar{X}$ monitoring schemes with control limits based on Equation (1) to monitor the process mean for normally distributed data. Thus, the schemes discussed herein will not outperform the more advanced schemes like the basic exponentially weighted moving average (EWMA) / cumulative 
sum (CUSUM), or the synthetic EWMA / CUSUM, or the runs-rules EWMA / CUSUM schemes. An interested reader may see Khoo et al. [14], Abbas et al. [1], [2], Riaz et al. [21], Scariano and Calzada [22] and Haq et al. [13]. Thus, this paper must be considered as a framework for quality practitioners who utilise Shewhart-type synthetic and runs-rules $\bar{X}$ charts. The rest of the paper is structured as follows: In Section 2 we present the operation of the MSS, the zero-state and steadystate Markov chain imbedding technique for the synthetic and runs-rules charts. In Section 3 we evaluate the OOC performance of the proposed MSS chart and compare its specific shift and overall performance with the Shewhart-type charts given in Table 1. In Section 4 we give an illustrative example using real-life data to show how these charts are implemented. Finally, in Section 5 we give concluding remarks.

\section{Operation and design considerations}

\subsection{Operation of the MSS chart}

The MSS synthetic $\bar{X}$ chart signals when two nonconforming samples out of $H+1$ successive samples plot above (below) the $U C L(L C L)$ which are separated by at most by $H-1$ conforming samples plotting between the $C L$ and the $U C L(L C L)$, respectively. This means that the MSS chart signals when all the $H+1$ consecutive samples that lead to an OOC event fall on one side of the $C L$. With the aid of definitions in Machado and Costa [19], to clearly describe the operation of the MSS scheme, we need to define two types of $C R L$ s i.e. lower $C R L$ (denoted by $C R L_{\mathrm{L}}$ ) and upper $C R L$ (denoted by $C R L_{\mathrm{U}}$ ). A $C R L_{\mathrm{L}}$ is the number conforming samples (i.e. falling on region $\mathrm{C}$ in Figure 1(c)) that are plotted in between the two consecutive nonconforming samples below the $L C L$, inclusive of the nonconforming sample at the end. However, a $C R L_{U}$ is the number conforming samples (i.e. falling on region B in Figure 1(c)) that are plotted in between the two consecutive nonconforming samples above the $U C L$, inclusive of the nonconforming sample at the end. Note that the absence of a conforming sample implies that either the $C R L_{\mathrm{U}}$ or $C R L_{\mathrm{L}}$ equals one. Thus the MSS chart operates as follows: 
Step (i) On the next sampling point, take a sample of size $n$ and compute $\bar{X}_{i}$.

Step (ii) If $L C L<\bar{X}_{i}<U C L$ then return to Step (i).

Step (iii) However, if $\bar{X}_{i} \leq L C L$ go to Step (iv), or $\bar{X}_{i} \geq U C L$ go to Step (v).

Step (iv) If $C R L_{\mathrm{L}} \leq H$ go to Step (vi), otherwise return to Step (i).

Step (v) If $C R L_{\mathrm{U}} \leq H$ go to Step (vi), otherwise return to Step (i).

Step (vi) Issue an OOC signal, and then take necessary corrective action to find and remove the assignable causes. Then return to Step (i).

\subsection{Markov chain imbedding techniques for the runs-type charts}

The transition probability matrix (TPM) of the Markov chain for any general (integer) value of $M>0$ is given by

$$
\boldsymbol{P}_{(M+1) \times(M+1)}=\left(\begin{array}{ccc}
\mathbf{Q}_{(M \times M)} & \mid & \boldsymbol{r}_{(M \times 1)} \\
- & - & - \\
\mathbf{0}_{(1 \times M)}^{\prime} & \mid & 1_{(1 \times 1)}
\end{array}\right)
$$

where $\mathbf{Q}_{(M \times M)}$ is the essential TPM, the vector $\mathbf{r}_{(M \times 1)}$ satisfies $\mathbf{r}=\mathbf{1}-\mathbf{Q 1}$ with $\mathbf{1}_{(M \times 1)}=$ $\left(\begin{array}{llll}1 & 1 & \ldots & 1\end{array}\right)^{T}$ and $\mathbf{0}_{(M \times 1)}=\left(\begin{array}{llll}0 & 0 & \ldots & 0\end{array}\right)^{T}$. In order to construct the TPM, we follow the Markov chain imbedding technique discussed briefly by Antzoulakos and Rakitzis [4], [5], Low et al. [17] and in detail by $\mathrm{Fu}$ and Lou [12]. This entails dividing the chart into separate distinct regions (see Figure 1) i.e. let $\left\{\bar{X}_{i} ; i \geq 1\right\}$ be a sequence of iid trials taking values in the set $\zeta_{1}=\{\mathrm{O}, \mathrm{U}\}, \zeta_{2}=\{\mathrm{A}, \mathrm{O}, \mathrm{D}\}$ and $\zeta_{3}=\{\mathrm{A}, \mathrm{B}, \mathrm{C}, \mathrm{D}\}$. Then, define the probability that a plotting statistic falls in each region:

(i) $\theta_{A}$ denotes the probability that a point falls above the $U C L$ (region A) i.e. $P\left(\bar{X}_{i} \in A\right)$;

(ii) $\theta_{B}$ denotes the probability that a point falls between the $C L$ and the $U C L$ (region B) i.e. $P\left(\bar{X}_{i} \in B\right)$

(iii) $\theta_{C}$ denotes the probability that a point falls between the $L C L$ and the $C L$ (region C) i.e. $P\left(\bar{X}_{i} \in C\right)$

(iv) $\theta_{D}$ denotes the probability that a point falls below the $L C L$ (region D) i.e. $P\left(\bar{X}_{i} \in D\right)$;

(v) $\theta_{O}=\theta_{B}+\theta_{C}$ denotes the probability that a point falls on region $\mathrm{O}$ i.e. $P\left(\bar{X}_{i} \in O\right)$;

(vi) $\theta_{U}=\theta_{A}+\theta_{D}$ denotes the probability that a point falls on region $\mathrm{U}$ i.e. $P\left(\bar{X}_{i} \in U\right)$. 
For some sample size, $n$, suppose that the values of $\mu_{\bar{X}}$ and $\sigma_{\bar{X}}^{2}$ are known. Thus the probabilities of a plotting statistic falling in a specific region are given by

$$
\begin{gathered}
\theta_{A}(\delta)=P(\bar{X} \geq U C L)=1-\Phi(k-\delta \sqrt{n}) \\
\theta_{B}(\delta)=P(C L \leq \bar{X}<U C L)=\Phi(k-\delta \sqrt{n})-\Phi(-\delta \sqrt{n}) \\
\theta_{C}(\delta)=P(L C L<\bar{X} \leq C L)=\Phi(-\delta \sqrt{n})-\Phi(-k-\delta \sqrt{n}) \\
\theta_{D}(\delta)=P(\bar{X} \leq L C L)=\Phi(-k-\delta \sqrt{n}) \\
\theta_{O}(\delta)=P(L C L<\bar{X}<U C L)=\theta_{B}(\delta)+\theta_{C}(\delta) \\
\theta_{U}(\delta)=P(\bar{X} \geq U C L)+P(\bar{X} \leq L C L)=\theta_{A}(\delta)+\theta_{D}(\delta)
\end{gathered}
$$

respectively, where $\Phi(\cdot)$ denotes the cumulative distribution function (cdf) of the standard normal distribution and $\delta$ is the shift parameter expressed in terms of the standard deviation units and we let $C L=0$.

To construct the TPM, we need to define the compound patterns that result in an OOC event (which is also known as the waiting time until the first occurrence of an OOC signal). For example, the sequence of plotting statistics 'AA' indicates two consecutive plotting statistics falling in region $\mathrm{A}$, whereas 'ABA' indicates the first plotting statistic falling in region $\mathrm{A}$, the second in region $\mathrm{B}$ and the third in region A, etc. The symbol ' \pm ' is used to denote the assumption that (at time 0 ) the first observation lies either above $U C L$ or below $L C L$ (i.e. HS feature), so that ' $\pm \mathrm{A}$ ' indicate the first plotting statistic falling either above $U C L$ (region A) or below $L C L$ (region D) and the second in region A. Following $\mathrm{Fu}$ and Lou [12], we let the sequences of conforming and nonconforming samples, say $\Lambda_{j}=\mathrm{ABBA}$, to be the $\mathrm{j}^{\text {th }}$ simple pattern within a sequence of $n$ four-state trials from say, set $\zeta_{3}$. Then, define $\Lambda$ as a compound pattern if it is the union of $\omega$ distinct simple patterns i.e. $\Lambda=\Lambda_{1} \cup \Lambda_{2} \cup \ldots \cup \Lambda_{\omega}$. Let $W$ denote the waiting time for the first occurrence of $\Lambda$. Then the runlength distribution of a control chart coincides with the waiting time distribution of $W$, see Antzoulakos and Rakitzis [4], [5]. That is, the run-length distribution of the chart becomes the waiting time until the first occurrence of one of the patterns $\Lambda_{1}, \Lambda_{2}, \ldots, \Lambda_{\omega}$ and these are the absorbing states of the Markov chain, where $\omega$ denotes the number of patterns (or sequences) of the 
$\bar{X}_{i}$ that cause the chart to signal. Then we define the Markov chain with the state space $\Omega=\{\phi$; $\eta_{1}, \ldots, \eta_{\tau}$; OOC $\}$ operating on $\left\{\bar{X}_{i} ; i \geq 1\right\}$ as follows:

- the transient state - corresponding to the IC region, denoted by $\phi$;

- the absorbing state - corresponding to the union of $\Lambda_{1}, \ldots, \Lambda_{\omega}$; in order to reduce the dimension of the TPM, the $\omega$ absorbing states which signal the entrance of the MC to each of the $\omega$ distinct simple patterns may be substituted by a single absorbing state, denoted by OOC;

- the sub-patterns - corresponding to the first element(s) of each of the simple pattern $\Lambda_{1}, \ldots$, $\Lambda_{\omega}$ without the last element. Note that these sub-patterns are non-absorbing and are denoted by $\eta_{1}, \ldots, \eta_{\tau}$, where $\tau \leq \omega$.

Table 2: Compound patterns of the Markov chain imbedding technique for the 2-of- $(H+1)$ synthetic and runs-rules charts when $H \leq 5$

\begin{tabular}{|c|c|c|c|c|}
\hline$H$ & DR / WS & KL / DW & $\mathrm{MC} 1 / \mathrm{MC} 2$ & $\mathrm{AR} / \mathrm{MSS}$ \\
\hline 1 & UU & $\mathrm{AA}, \pm \mathbf{A}, \mathrm{DD}, \pm \mathbf{D}$ & $\mathrm{AA}, \pm \mathbf{A}, \mathrm{DD}, \pm \mathbf{D}$ & $\mathrm{AA}, \pm \mathbf{A}, \mathrm{DD}, \pm \mathbf{D}$ \\
\hline 2 & UOU & $\mathrm{AOA}, \mathrm{ADA}, \pm \mathbf{O A}, \mathrm{DOD}, \mathrm{DAD}, \pm \mathbf{O D}$ & $\mathrm{AOA}, \pm \mathbf{O A}, \mathrm{DOD}, \pm \mathbf{O D}$ & $\mathrm{ABA}, \pm \mathbf{B A}, \mathrm{DCD}, \pm \mathbf{C D}$ \\
\hline 3 & UOOU & $\begin{array}{l}\text { AOOA, AODA, ADOA, } \pm \text { OOOA, } \\
\text { DOOD, DOAD, DAOD } \pm \text { } \pm \text { OOD }\end{array}$ & $\begin{array}{l}\text { AOOA, } \pm \text { OOOA, } \\
\text { DOOD }, \pm \text { OOD }\end{array}$ & $\begin{array}{l}\mathrm{ABBA}, \pm \mathbf{B B A} \\
\mathrm{DCCD}, \pm \mathbf{C C D}\end{array}$ \\
\hline 4 & UOOOU & $\begin{array}{l}\text { AOOOA, AOODA, AODOA, ADOOA, } \pm \text { OOOA, } \\
\text { DOOOD, DOOAD, DOAOD, DAOOD } \pm \text { OOOOD }\end{array}$ & $\begin{array}{l}\text { AOOOA, } \pm \text { OOOA, } \\
\text { DOOOD }, \pm \text { OOOD }\end{array}$ & $\begin{array}{l}\mathrm{ABBBA}, \pm \mathbf{B B B A} \\
\mathrm{DCCCD}, \pm \mathbf{C C C D}\end{array}$ \\
\hline 5 & UOOOOU & $\begin{array}{l}\text { AOOOOA, AOOODA, AOODOA, AODOOA, } \\
\text { ADOOOA, } \pm \text { OOOOA, } \\
\text { DOOOOD, DOOOAD, DOOAOD, DOAOOD, } \\
\text { DAOOOD, } \pm \text { OOOOD }\end{array}$ & $\begin{array}{l}\text { AOOOOA, } \pm \text { OOOOOA, } \\
\text { DOOOOD }, \pm \text { OOOOD }\end{array}$ & $\begin{array}{l}\text { ABBBBA, } \mathbf{\pm B B B B A} \\
\text { DCCCCD }, \pm \mathbf{C C C C D}\end{array}$ \\
\hline
\end{tabular}

For illustration purpose, in Table 2 we give the compound patterns of $H \leq 5$ for the eight schemes in Table 1 with charting regions in Figure 1. The compound patterns of KL, MC1 and AR schemes exclude those simple patterns that are boldfaced in Table 2 (i.e. those starting with ' \pm '). The aim is to show how to construct the TPM in Equation (2) and more importantly to show the difference between TPMs of DR and WS, KL and DW, MC1 and MC2, AR and MSS, respectively, that is, by removing the HS feature elements (or simple / sub-patterns) in the construction of the TPMs of the DW, MC2 and MSS results in the TPMs of KL, MC1 and AR, respectively. Note that the WS chart is non-side-sensitive, thus the initial state at time zero is in the nonconforming region 
U. Next, we show how to construct the state space $\Omega$ using an example with $H=2$. The state spaces of the eight different monitoring schemes listed in Table 1 when $H=2$ are given by:

- transient state

DR, KL, MC1, WS, DW, MC2: $\phi=\{\mathrm{O}\} ; \mathrm{AR}, \mathrm{MSS}: \phi=\{\mathrm{B}, \mathrm{C}\}$; see Figure 1.

- absorbing state

These are the union of the simple patterns listed in Table 2 corresponding to $H \leq 2$ as these yield an observed $C R L \leq 2$ for the WS, DW and MC2 schemes; and $C R L_{\mathrm{L}} \leq 2$ or $C R L_{\mathrm{U}} \leq 2$ for the MSS scheme.

DR: $\Lambda_{1}=\{\mathrm{UU}\}, \Lambda_{2}=\{\mathrm{UOU}\}$, i.e. $\omega=2$.

$\mathrm{KL}: \Lambda_{1}=\{\mathrm{ADA}\}, \Lambda_{2}=\{\mathrm{AOA}\}, \Lambda_{3}=\{\mathrm{AA}\}, \Lambda_{4}=\{\mathrm{DD}\}, \Lambda_{5}=\{\mathrm{DOD}\}, \Lambda_{6}=\{\mathrm{DAD}\}$, i.e. $\omega=6$;

$\mathrm{MC} 1: \Lambda_{1}=\{\mathrm{AOA}\}, \Lambda_{2}=\{\mathrm{AA}\}, \Lambda_{3}=\{\mathrm{DD}\}, \Lambda_{4}=\{\mathrm{DOD}\}$, i.e. $\omega=4$

$\mathrm{AR}: \Lambda_{1}=\{\mathrm{ABA}\}, \Lambda_{2}=\{\mathrm{AA}\}, \Lambda_{3}=\{\mathrm{DD}\}, \Lambda_{4}=\{\mathrm{DCD}\}$, i.e. $\omega=4$

WS: $\Lambda_{1}=\{\mathrm{UU}\}, \Lambda_{2}=\{\mathrm{UOU}\}$, i.e. $\omega=2$;

DW: $\Lambda_{1}=\{\mathrm{ADA}\}, \Lambda_{2}=\{\mathrm{AOA}\}, \Lambda_{3}=\{\mathrm{AA}\}, \Lambda_{4}=\{\mathrm{DD}\}, \quad \Lambda_{5}=\{\mathrm{DOD}\}, \Lambda_{6}=\{\mathrm{DAD}\}, \Lambda_{7}=\{ \pm \mathrm{A}\}$, $\Lambda_{8}=\{ \pm \mathrm{D}\}, \Lambda_{9}=\{ \pm \mathrm{OA}\}, \Lambda_{10}=\{ \pm \mathrm{OD}\}$, i.e. $\omega=10$

MC2: $\Lambda_{1}=\{\mathrm{AOA}\}, \quad \Lambda_{2}=\{\mathrm{AA}\}, \quad \Lambda_{3}=\{\mathrm{DD}\}, \quad \Lambda_{4}=\{\mathrm{DOD}\}, \quad \Lambda_{5}=\{ \pm \mathrm{A}\}, \quad \Lambda_{6}=\{ \pm \mathrm{D}\}, \quad \Lambda_{7}=\{ \pm \mathrm{OA}\}$, $\Lambda_{8}=\{ \pm \mathrm{OD}\}$, i.e. $\omega=8$

MSS: $\Lambda_{1}=\{\mathrm{ABA}\}, \quad \Lambda_{2}=\{\mathrm{AA}\}, \quad \Lambda_{3}=\{\mathrm{DD}\}, \quad \Lambda_{4}=\{\mathrm{DCD}\}, \quad \Lambda_{5}=\{ \pm \mathrm{A}\}, \quad \Lambda_{6}=\{ \pm \mathrm{D}\}, \quad \Lambda_{7}=\{ \pm \mathrm{BA}\}$, $\Lambda_{8}=\{ \pm \mathrm{CD}\}$, i.e. $\omega=8$;

- $\quad$ sub-patterns - these are distinct sub-patterns of $\Lambda_{1}, \ldots, \Lambda_{\omega}$

DR: $\eta_{1}=\{\mathrm{U}\}, \eta_{2}=\{\mathrm{UO}\}$, i.e. $\tau=2$.

$\mathrm{KL}: \eta_{1}=\{\mathrm{AD}\}, \eta_{2}=\{\mathrm{AO}\}, \eta_{3}=\{\mathrm{A}\}, \eta_{4}=\{\mathrm{D}\}, \eta_{5}=\{\mathrm{DO}\}, \eta_{6}=\{\mathrm{DA}\}$, i.e. $\tau=6$;

$\mathrm{MC} 1: \eta_{1}=\{\mathrm{AO}\}, \eta_{2}=\{\mathrm{A}\}, \eta_{3}=\{\mathrm{D}\}, \eta_{4}=\{\mathrm{DO}\}$, i.e. $\tau=4$

AR: $\eta_{1}=\{\mathrm{AB}\}, \eta_{2}=\{\mathrm{A}\}, \eta_{3}=\{\mathrm{D}\}, \eta_{4}=\{\mathrm{DC}\}$, i.e. $\tau=4$

WS: $\eta_{1}=\{\mathrm{U}\}, \eta_{2}=\{\mathrm{UO}\}$, i.e. $\tau=2$;

DW: $\eta_{1}=\{\mathrm{AD}\}, \eta_{2}=\{\mathrm{AO}\}, \eta_{3}=\{\mathrm{A}\}, \eta_{4}=\{\mathrm{D}\}, \eta_{5}=\{\mathrm{DO}\}, \eta_{6}=\{\mathrm{DA}\}, \eta_{7}=\{ \pm\}, \eta_{8}=\{ \pm \mathrm{O}\}$, i.e. $\tau=8$; 
MC2: $\eta_{1}=\{\mathrm{AO}\}, \eta_{2}=\{\mathrm{A}\}, \eta_{3}=\{\mathrm{D}\}, \eta_{4}=\{\mathrm{DO}\}, \eta_{5}=\{ \pm\}, \eta_{6}=\{ \pm \mathrm{O}\}$, i.e. $\tau=6$;

MSS: $\eta_{1}=\{\mathrm{AB}\}, \eta_{2}=\{\mathrm{A}\}, \eta_{3}=\{\mathrm{D}\}, \eta_{4}=\{\mathrm{DC}\}, \eta_{5}=\{ \pm\}, \eta_{6}=\{ \pm \mathrm{B}\}, \eta_{7}=\{ \pm \mathrm{C}\}$, i.e. $\tau=7$

Thus, when $H=2$ the TPM of the WS, DW, MC2 and MSS schemes are given by

\begin{tabular}{c|cccc|} 
& $\phi$ & $\eta_{1}$ & $\eta_{2}$ & OOC \\
\hline$\phi$ & $\theta_{O}$ & $1-\theta_{O}$ & 0 & 0 \\
$\eta_{1}$ & 0 & 0 & $\theta_{O}$ & $1-\theta_{O}$ \\
$\eta_{2}$ & $\theta_{O}$ & 0 & 0 & $1-\theta_{O}$ \\
OOC & 0 & 0 & 0 & 1 \\
\cline { 2 - 6 }
\end{tabular}

\begin{tabular}{c|cccccccccc|} 
& $\eta_{1}$ & $\eta_{2}$ & $\eta_{3}$ & $\phi$ & $\eta_{4}$ & $\eta_{5}$ & $\eta_{6}$ & $\eta_{7}$ & $\eta_{8}$ & OOC \\
\hline$\eta_{1}$ & 0 & 0 & 0 & 0 & 0 & $\theta_{O}$ & 0 & 0 & 0 & $\theta_{A}+\theta_{D}$ \\
$\eta_{2}$ & 0 & 0 & 0 & $\theta_{O}$ & $\theta_{D}$ & 0 & 0 & 0 & 0 & $\theta_{A}$ \\
$\eta_{3}$ & $\theta_{D}$ & $\theta_{O}$ & 0 & 0 & 0 & 0 & 0 & 0 & 0 & $\theta_{A}$ \\
$\phi$ & 0 & 0 & $\theta_{A}$ & $\theta_{O}$ & $\theta_{D}$ & 0 & 0 & 0 & 0 & 0 \\
$\eta_{4}$ & 0 & 0 & 0 & 0 & 0 & $\theta_{O}$ & $\theta_{A}$ & 0 & 0 & $\theta_{D}$ \\
$\eta_{5}$ & 0 & 0 & $\theta_{A}$ & $\theta_{O}$ & 0 & 0 & 0 & 0 & 0 & $\theta_{D}$ \\
$\eta_{6}$ & 0 & $\theta_{O}$ & 0 & 0 & 0 & 0 & 0 & 0 & 0 & $\theta_{A}+\theta_{D}$ \\
$\eta_{7}$ & 0 & 0 & 0 & 0 & 0 & 0 & 0 & 0 & $\theta_{O}$ & $\theta_{A}+\theta_{D}$ \\
$\eta_{8}$ & 0 & 0 & 0 & $\theta_{O}$ & 0 & 0 & 0 & 0 & 0 & $\theta_{A}+\theta_{D}$ \\
OOC & 0 & 0 & 0 & 0 & 0 & 0 & 0 & 0 & 0 & 1 \\
\hline
\end{tabular}

\begin{tabular}{c|cccccccc|} 
& $\eta_{1}$ & $\eta_{2}$ & $\phi$ & $\eta_{3}$ & $\eta_{4}$ & $\eta_{5}$ & $\eta_{6}$ & OOC \\
\hline$\eta_{1}$ & 0 & 0 & $\theta_{O}$ & $\theta_{D}$ & 0 & 0 & 0 & $\theta_{A}$ \\
$\eta_{2}$ & $\theta_{O}$ & 0 & 0 & $\theta_{D}$ & 0 & 0 & 0 & $\theta_{A}$ \\
$\phi$ & 0 & $\theta_{A}$ & $\theta_{O}$ & $\theta_{D}$ & 0 & 0 & 0 & 0 \\
$\eta_{3}$ & 0 & $\theta_{A}$ & 0 & 0 & $\theta_{O}$ & 0 & 0 & $\theta_{D}$ \\
$\eta_{4}$ & 0 & $\theta_{A}$ & $\theta_{O}$ & 0 & 0 & 0 & 0 & $\theta_{D}$ \\
$\eta_{5}$ & 0 & 0 & 0 & 0 & 0 & 0 & $\theta_{O}$ & $\theta_{A}+\theta_{D}$ \\
$\eta_{6}$ & 0 & 0 & $\theta_{O}$ & 0 & 0 & 0 & 0 & $\theta_{A}+\theta_{D}$ \\
OOC & 0 & 0 & 0 & 0 & 0 & 0 & 0 & 1 \\
\hline
\end{tabular}

and

\begin{tabular}{c|ccccccccc} 
& $\eta_{1}$ & $\eta_{2}$ & $\phi$ & $\eta_{3}$ & $\eta_{4}$ & $\eta_{5}$ & $\eta_{6}$ & $\eta_{7}$ & OOC \\
\hline$\eta_{1}$ & 0 & 0 & $\theta_{B}+\theta_{C}$ & $\theta_{D}$ & 0 & 0 & 0 & 0 & $\theta_{A}$ \\
$\eta_{2}$ & $\theta_{B}$ & 0 & $\theta_{C}$ & $\theta_{D}$ & 0 & 0 & 0 & 0 & $\theta_{A}$ \\
$\phi$ & 0 & $\theta_{A}$ & $\theta_{B}+\theta_{C}$ & $\theta_{D}$ & 0 & 0 & 0 & 0 & 0 \\
$\eta_{3}$ & 0 & $\theta_{A}$ & $\theta_{B}$ & 0 & $\theta_{C}$ & 0 & 0 & 0 & $\theta_{D}$ \\
$\eta_{4}$ & 0 & $\theta_{A}$ & $\theta_{B}+\theta_{C}$ & 0 & 0 & 0 & 0 & 0 & $\theta_{D}$ \\
$\eta_{5}$ & 0 & 0 & 0 & 0 & 0 & 0 & $\theta_{B}$ & $\theta_{C}$ & $\theta_{A}+\theta_{D}$ \\
$\eta_{6}$ & 0 & 0 & $\theta_{B}+\theta_{C}$ & $\theta_{D}$ & 0 & 0 & 0 & 0 & $\theta_{A}$ \\
$\eta_{7}$ & 0 & $\theta_{A}$ & $\theta_{B}+\theta_{C}$ & 0 & 0 & 0 & 0 & 0 & $\theta_{D}$ \\
OOC & 0 & 0 & 0 & 0 & 0 & 0 & 0 & 0 & 1
\end{tabular}


respectively. Moreover, the TPM of the DR scheme is the same as that of WS. However, the TPM of the KL, MC1 and AR are obtained from those of DW, MC2 and MSS schemes by removing the elements with the HS feature (i.e. $\eta_{7}$ and $\eta_{8}$ for DW scheme; $\eta_{5}$ and $\eta_{6}$ for MC2 scheme; $\eta_{5}, \eta_{6}$ and $\eta_{7}$ for MSS scheme) in Equations (5), (6) and (7), respectively; note that this relation holds for every $H>0$. The construction of the Markov chain TPMs follows in a similar manner for other values of $H$. Finally, for any $H>0$, the dimension (i.e. $M+1$ ) of the TPM in Equation (2) for the runs-rules and synthetic charts are given by

$$
\text { DR: }(H+1)+1, \quad \mathrm{KL}:\left(H^{2}+H+1\right)+1, \mathrm{MC} 1:(2 H+1)+1, \mathrm{AR}:(2 H+1)+1
$$

and

WS: $(H+1)+1, \quad \mathrm{DW}:\left[\left(H^{2}+H+1\right)+H\right]+1, \mathrm{MC} 2:[(2 H+1)+H]+1, \quad \mathrm{MSS}:[(2 H+1)+(2 H-1)]+1$, respectively.

\subsection{Design considerations}

The performance of a control chart at some specific shift is usually measured by $A R L$ given by

$$
A R L(\delta)=\xi(\mathbf{I}-\mathbf{Q}(\delta))^{-1} \mathbf{1}
$$

where $\xi$ is the initial probability vector (depending on whether a zero-state or a steady-state analysis is being considered), see Fu and Lou [12]. Zero-state and steady-state analysis are used to characterize short and long term run-length properties of a control chart, respectively. That is, the zero-state run-length is the number of sampling points at which the chart first signals given it begins in some initial state, however, the steady-state run-length is the number of sampling points at which the chart first signals given that the process begins and stays IC for a very long time, then at some random time, an OOC signal is observed, see Champ [8] and Machado and Costa [18], [19]. Note that $\mathbf{q}_{(1 \times M)}$ is the row vector of initial probabilities associated with the zero-state mode i.e.

$$
\xi=\mathbf{q}_{(1 \times M)}=\left(\begin{array}{lllll}
0 & 0 & \ldots & \ldots & \ldots
\end{array}\right)
$$

so that the initial state element on the TPM corresponds to the value of 1 in Equation (10). That is, it has a one in the component associated with the state in which the chart begins and each of the other 
components is zero. For the MSS, MC2 and DW charts, the initial state corresponds to the element of the TPM equal to ' \pm ', whereas for WS, it corresponds to the element with ' $U$ '. However, for AR it corresponds to 'B,C' and for $\mathrm{MC} 1, \mathrm{KL}$ and $\mathrm{DR}$, it corresponds to ' $\mathrm{O}$ ', that is, for runs-rules without HS, the initial state is given by ' $\phi$ '. In steady-state, the vector $\mathbf{q}$ is replaced by a vector $\mathbf{s}$, i.e. the steady-state initial probability vector given by

$$
\xi=\mathbf{s}_{(1 \times M)}=\left(s_{1} s_{2} \ldots s_{M}\right)
$$

where the sum of the elements in Equation (11) sum to unity. Champ [8] showed that when $\mathbf{Q}_{\mathbf{0}}$ is obtained from $\mathbf{Q}(0)$ (i.e. $\delta=0$ ) after diving each element by its corresponding row sum, then $\mathbf{s}_{(1 \times M)}$ is a vector such that $\mathbf{s}=\mathbf{s} \cdot \mathbf{Q}_{\mathbf{0}}$ subject to $\sum_{i=1}^{M} s_{i}=1$. The $A R L$ (in Equation (9)) based on Equation (10) is denoted by ZSARL, whereas that based on Equation (11) is denoted by SSARL.

Since the $A R L$ only measures the performance of a control chart at some specific shifts, a number of authors have suggested the use of additional indices to measure the overall performance of the charts, see for example Wu et al. [25], Abujiya et al. [3] and Machado and Costa [19]. Since it is usually unknown what specific shift value(s) a control chart should be optimized for, Wu et al. [25] stated that it is more efficient to design a chart such that it has a better overall performance than its competitors. Thus, when the aim is to measure the overall performance of the chart over a range of shifts (i.e. $0<\delta \leq \delta_{\max }$, where $\delta_{\max }$ is the upper bound of the mean shift that is of interest by the user), the objective function must be defined in terms of the extra quadratic loss $(E Q L)$ i.e.

$$
E Q L=\frac{1}{\delta_{\max }} \int_{0}^{\delta_{\max }} \delta^{2} \times A R L(\delta) d \delta=\text { minimum. }
$$

Since it is generally assumed that all mean shifts within the range $0<\delta \leq \delta_{\max }$ occur with equal probability, a uniform distribution of $\delta$ is implied, see Wu et al. [25] and Machado and Costa [19]. Hence Equation (12) may equivalently be written as

$$
E Q L=\frac{1}{\delta_{\max }} \sum_{0}^{\delta_{\max }} \delta^{2} \times A R L(\delta)=\text { minimum }
$$


Here, we use a step shift $(\delta)$ of size 0.1 . In addition to the $E Q L, \mathrm{Wu}$ et al. [25] suggested the performance comparison index $(P C I)$ to measure the relative effectiveness of two different charts. In this paper, we shall use the MSS chart as the benchmark; hence the PCI is given by

$$
P C I=\frac{E Q L}{E Q L_{M S S}}
$$

where $E Q L_{\mathrm{MSS}}$ is $E Q L$ of the 'benchmark' MSS chart. Also, the ratio of the $A R L$ s is usually used to measure the overall effectiveness of a benchmark chart against other competitors, see $\mathrm{Wu}$ et al. [25]. Hence assuming a uniform distribution in $\delta$, the average ratio of $A R L$ s is given by

$$
A R A R L=\frac{1}{\delta_{\max }} \sum_{0}^{\delta_{\max }} \frac{A R L(\delta)}{A R L_{M S S}(\delta)}
$$

where $A R L_{\mathrm{MSS}}(\delta)$ is the $A R L$ produced by the MSS chart. If the value of $P C I$ or $A R A R L$ is larger than one, the competing chart will produce larger OOC $A R L$ over a larger shift range and / or to a larger degree compared to the benchmark chart and thus, the competing chart is relatively less effective. However, if the $P C I$ or $A R A R L$ is smaller than one, the competing chart will have higher overall effectiveness than the benchmark chart.

\section{Discussion}

In this section, we conduct an empirical study of the MSS scheme and compare its performance to the schemes listed in Table 1 for the zero-state and steady-state mode. Firstly, though in Table 3, we use Equations (2), (3), (8a), (8b), (9) in conjunction with Equations (10) and (11) to determine the optimal values of $k$ in both states when $H=1,2, \ldots, 20$ for $A R L_{0}=200$, 370.4, 500 and 1000.

It is very interesting to notice that as $H$ increases, the optimal values of $k$ also increases for all the charts considered in Table 3, however, the newly proposed MSS scheme (and its runs-rules counterpart AR) produces values of $k$ that converges to some specific value regardless of how large $H$ is, for both zero-state and steady-state mode. In this section, we investigate what implications does this convergence has on the overall performance, as $H$ increases. Firstly though, we conduct an 
Table 3: The values of $k$ for the zero-state and steady-state 2 -of- $(H+1)$ synthetic and runs-rules $\bar{X}$ charts when $H=1,2, \ldots, 20$ and $A R L_{0}=200,370.4,500,1000$

\begin{tabular}{|c|c|c|c|c|c|c|c|c|c|c|c|c|c|c|c|c|c|c|c|c|c|c|c|c|}
\hline & \multicolumn{12}{|c|}{ (a) $A R L_{0}=\mathbf{2 0 0}$} & \multicolumn{12}{|c|}{ (b) $A R L_{0}=\mathbf{3 7 0 . 4}$} \\
\hline & \multicolumn{8}{|c|}{ Zero-state } & \multicolumn{4}{|c|}{$\begin{array}{l}\text { Steady-state } \\
\text { St }\end{array}$} & \multicolumn{8}{|c|}{ Zero-state } & \multicolumn{4}{|c|}{ Steady-state } \\
\hline $\mathrm{H}$ & DR & KL & $\mathrm{MC} 1$ & AR & WS & DW & MC2 & MSS & $\begin{array}{c}\text { DR\& } \\
\text { WS }\end{array}$ & $\begin{array}{l}\text { KL\& } \\
\text { DW }\end{array}$ & $\begin{array}{c}\text { MC1\& } \\
\text { MC2 }\end{array}$ & $\begin{array}{l}\text { AR\& } \\
\text { MSS }\end{array}$ & DR & KL & $\mathrm{MC1}$ & AR & WS & DW & MC2 & MSS & $\begin{array}{c}\text { DR\& } \\
\text { WS }\end{array}$ & $\begin{array}{c}\text { KL\& } \\
\text { DW }\end{array}$ & $\begin{array}{c}\text { MC1\& } \\
\text { MC2 }\end{array}$ & $\begin{array}{l}\text { AR\& } \\
\text { MSS }\end{array}$ \\
\hline 1 & 1.7912 & 1.6327 & 1.6327 & 1.6327 & 1.8073 & 1.6569 & 1.6569 & 1.6569 & 1.7923 & 1.6339 & 1.6339 & 1.6339 & 1.9323 & 1.7814 & 1.7814 & 1.7814 & 1.9435 & 1.7982 & 1.7982 & 1.7982 & 1.9328 & 1.7820 & 1.7820 & 1.7820 \\
\hline 2 & 1.9330 & 1.7870 & 1.7828 & 1.7203 & 1.9545 & 1.8186 & 1.8151 & 1.7489 & 1.9345 & 1.7888 & 1.7845 & 1.7219 & 2.0698 & 1.9293 & 1.9265 & 1.8664 & 2.0848 & 1.9515 & 1.9489 & 1.8862 & 2.0706 & 1.9303 & 1.9274 & 1.8671 \\
\hline 3 & 2.0110 & 1.8718 & 1.8654 & 1.7514 & 2.0366 & 1.9088 & 1.9035 & 1.7819 & 2.0130 & 1.8740 & 1.8675 & 1.7532 & 2.1461 & 2.0113 & 2.0069 & 1.8969 & 2.1640 & 2.0374 & 2.0335 & 1.9181 & 2.1472 & 2.0125 & 2.0080 & 1.8978 \\
\hline 4 & 2.0643 & 1.9296 & 1.9216 & 1.7644 & 2.0932 & 1.9711 & 1.9646 & 1.7958 & 2.0667 & 1.9323 & 1.9242 & 1.7663 & 2.1985 & 2.0676 & 2.0619 & 1.9099 & 2.2188 & 2.0968 & 2.0920 & 1.9318 & 2.1997 & 2.0689 & 2.0633 & 1.9109 \\
\hline 5 & 2.1043 & 1.9731 & 1.9638 & 1.7702 & 2.1361 & 2.0185 & 2.0111 & 1.8020 & 2.1071 & 1.9762 & 1.9668 & 1.7722 & 2.2380 & 2.1101 & 2.1035 & 1.9158 & 2.2604 & 2.1421 & 2.1366 & 1.9380 & 2.2395 & 2.1117 & 2.1051 & 1.9169 \\
\hline 6 & 2.1361 & 2.0078 & 1.9973 & 1.7728 & 2.1705 & 2.0566 & 2.0485 & 1.8049 & 2.1393 & 2.0113 & 2.0008 & 1.7749 & 2.2697 & 2.1441 & 2.1367 & 1.9186 & 2.2939 & 2.1787 & 2.1726 & 1.9409 & 2.2714 & 2.1459 & 2.1385 & 1.9197 \\
\hline 7 & 2.1624 & 2.0395 & 2.0250 & 1.7740 & 2.1992 & 2.0908 & 2.0797 & 1.8062 & 2.1661 & 2.0435 & 2.0290 & 1.7762 & 2.2959 & 2.1746 & 2.1642 & 1.9199 & 2.3218 & 2.2111 & 2.2026 & 1.9422 & 2.2978 & 2.1767 & 2.1663 & 1.9210 \\
\hline 8 & 2.1848 & 2.0609 & 2.0486 & 1.7746 & 2.2238 & 2.1157 & 2.1065 & 1.8068 & 2.1888 & 2.0652 & 2.0530 & 1.7767 & 2.3183 & 2.1964 & 2.1877 & 1.9205 & 2.3458 & 2.2354 & 2.2283 & 1.9429 & 2.3204 & 2.1987 & 2.1900 & 1.9216 \\
\hline 9 & 2.2041 & 2.0820 & 2.0689 & 1.7748 & 2.2452 & 2.1395 & 2.1299 & 1.8071 & 2.2085 & 2.0868 & 2.0737 & 1.7770 & 2.3377 & 2.2174 & 2.2081 & 1.9208 & 2.3667 & 2.2583 & 2.2509 & 1.9432 & 2.3401 & 2.2199 & 2.2106 & 1.9219 \\
\hline 10 & 2.2211 & 2.1006 & 2.0868 & 1.7750 & 2.2641 & 2.1607 & 2.1506 & 1.8072 & 2.2259 & 2.1058 & 2.0921 & 1.7771 & 2.3549 & 2.2359 & 2.2261 & 1.9209 & 2.3852 & 2.2786 & 2.2709 & 1.9433 & 2.3575 & 2.2386 & 2.2288 & 1.9220 \\
\hline 11 & 2.2363 & 2.1173 & 2.1027 & 1.7750 & 2.2811 & 2.1796 & 2.1693 & 1.8073 & 2.2415 & 2.1229 & 2.1084 & 1.7772 & 2.3702 & 2.2524 & 2.2422 & 1.9210 & 2.4018 & 2.2969 & 2.2889 & 1.9434 & 2.3730 & 2.2554 & 2.2451 & 1.9221 \\
\hline 12 & 2.2499 & 2.1322 & 2.1171 & 1.7751 & 2.2965 & 2.1968 & 2.1862 & 1.8073 & 2.2555 & 2.1382 & 2.1231 & 1.7772 & 2.3841 & 2.2674 & 2.2566 & 1.9210 & 2.4169 & 2.3135 & 2.3052 & 1.9435 & 2.3870 & 2.2706 & 2.2599 & 1.9221 \\
\hline 13 & 2.2623 & 2.1458 & 2.1300 & 1.7751 & 2.3105 & 2.2126 & 2.2016 & 1.8073 & 2.2682 & 2.1522 & 2.1365 & 1.7772 & 2.3966 & 2.2810 & 2.2698 & 1.9210 & 2.4307 & 2.3286 & 2.3201 & 1.9435 & 2.3998 & 2.2844 & 2.2733 & 1.9221 \\
\hline 14 & 2.2736 & 2.1582 & 2.1419 & 1.7751 & 2.3234 & 2.2270 & 2.2158 & 1.8073 & 2.2799 & 2.1650 & 2.1488 & 1.7772 & 2.4082 & 2.2935 & 2.2819 & 1.9210 & 2.4433 & 2.3426 & 2.3338 & 1.9435 & 2.4116 & 2.2971 & 2.2856 & 1.9221 \\
\hline 15 & 2.2840 & 2.1697 & 2.1528 & 1.7751 & 2.3353 & 2.2404 & 2.2290 & 1.8073 & 2.2906 & 2.1768 & 2.1601 & 1.7772 & 2.4188 & 2.3050 & 2.2930 & 1.9210 & 2.4551 & 2.3556 & 2.3466 & 1.9435 & 2.4224 & 2.3088 & 2.2969 & 1.9221 \\
\hline 16 & 2.2936 & 2.1802 & 2.1628 & 1.7751 & 2.3464 & 2.2529 & 2.2412 & 1.8073 & 2.3006 & 2.1878 & 2.1706 & 1.7772 & 2.4287 & 2.3157 & 2.3034 & 1.9210 & 2.4660 & 2.3676 & 2.3584 & 1.9435 & 2.4324 & 2.3197 & 2.3074 & 1.9221 \\
\hline 17 & 2.3025 & 2.1901 & 2.1722 & 1.7751 & 2.3567 & 2.2645 & 2.2527 & 1.8073 & 2.3099 & 2.1980 & 2.1803 & 1.7772 & 2.4378 & 2.3257 & 130 & 1.9210 & 2.4762 & 2.3789 & 2.3695 & 1.9435 & 2.4418 & 2.3299 & 2.3173 & 1.9221 \\
\hline 18 & 2.3108 & 2.1992 & 2.1809 & 1.7751 & 2.3664 & 2.2755 & 2.2634 & 1.8073 & 2.3185 & 2.2076 & 2.1894 & 1.7772 & 2.4464 & 2.3350 & 2.3219 & 1.9210 & 2.4857 & 2.3895 & 2.3799 & 1.9435 & 2.4506 & 2.3394 & 2.3264 & 1.9221 \\
\hline 19 & 2.3186 & 2.2078 & 2.1890 & 1.7751 & 2.3755 & 2.2858 & 2.2735 & 1.8073 & 2.3267 & 2.2166 & 2.1979 & 1.7772 & 2.4545 & 2.3437 & 2.3303 & 1.9210 & 2.4947 & 2.3995 & 2.3898 & 1.9435 & 2.4588 & 2.3437 & 2.3351 & 1.9221 \\
\hline \multirow[t]{3}{*}{20} & 2.3259 & 2.2159 & 2.1966 & 1.7751 & 2.3841 & 2.2955 & 2.2831 & 1.8073 & 2.3343 & 2.2250 & 2.2060 & 1.7772 & 2.4620 & 2.3520 & 2.3382 & 1.9210 & 2.5032 & 2.4089 & 2.3990 & 1.9435 & 2.4666 & 2.3568 & 2.3432 & 1.9221 \\
\hline & \multicolumn{12}{|c|}{ (c) $A R L_{0}=\mathbf{5 0 0}$} & \multicolumn{12}{|c|}{ (d) $A R L_{0}=\mathbf{1 0 0 0}$} \\
\hline & \multicolumn{8}{|c|}{ Zero-state } & \multicolumn{4}{|c|}{ Steady-state } & \multicolumn{8}{|c|}{ Zero-state } & \multicolumn{4}{|c|}{ Steady-state } \\
\hline $\mathrm{H}$ & DR & KL & MC1 & AR & WS & DW & MC2 & MSS & $\begin{array}{c}\text { DR\& } \\
\text { WS }\end{array}$ & $\begin{array}{c}\text { KL\& } \\
\text { DW }\end{array}$ & $\begin{array}{c}\text { MC1\& } \\
\text { MC2 }\end{array}$ & $\begin{array}{l}\text { AR\& } \\
\text { MSS } \\
\end{array}$ & DR & KL & MC1 & AR & WS & DW & MC2 & MSS & $\begin{array}{c}\text { DR\& } \\
\text { WS }\end{array}$ & $\begin{array}{c}\text { KL\& } \\
\text { DW }\end{array}$ & $\begin{array}{c}\text { MC1\& } \\
\text { MC2 }\end{array}$ & $\begin{array}{l}\text { AR\& } \\
\text { MSS } \\
\end{array}$ \\
\hline $\begin{array}{ll}1 \\
\end{array}$ & (1.9979 & $\begin{array}{l}1.8504 \\
\end{array}$ & $\begin{array}{ll}1.8504 \\
\end{array}$ & (1.8504 & 2.0073 & 8645 & "1.8645 & 1.8645 & $\begin{array}{l}1.9983 \\
\end{array}$ & 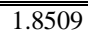 & $\begin{array}{ll}1.8509 \\
\end{array}$ & $\begin{array}{ll}1.8509 \\
\end{array}$ & 2.1428 & $\overline{.00}$ & 2.0026 & 2.0026 & 2. & - & $\begin{array}{c}2.0120 \\
\end{array}$ & 2.0120 & $\begin{array}{l}2.1430 \\
\end{array}$ & $\begin{array}{c}2.0028 \\
\end{array}$ & 2.0028 & 2.0028 \\
\hline 2 & 2.1333 & 1.9954 & 1.9930 & 1.9340 & 2.1459 & 2.0140 & 2.0119 & 1.9507 & 2.1339 & 1.9961 & 1.9937 & 1.9346 & 2.2735 & 2.1412 & 2.1396 & 2.0830 & 2.2820 & 2.1537 & 2.1522 & 2.0942 & 2.2738 & 2.1415 & 2.1399 & 2.0833 \\
\hline 3 & 2.2087 & 2.0760 & 2.0723 & 1.9642 & 2.2238 & 2.0980 & 2.0947 & 1.9821 & 2.2095 & 2.0769 & 2.0731 & 1.9649 & 2.3467 & 2.2188 & 2.2163 & 2.1125 & 2.3569 & 2.2336 & 2.2313 & 2.1244 & 2.3471 & 2.2192 & 2.2167 & 2.1128 \\
\hline 4 & 2.2606 & 2.1315 & 2.1267 & 1.9772 & 2.2777 & 2 & 2 & & 2.2615 & 2.1325 & & 1.9780 & 12 & 2.2 & & 2.1252 & & 2.2891 & 2.2862 & & & 2.2728 & & 2.1256 \\
\hline 5 & 2.2998 & 2.1734 & 2.1679 & 1.9832 & 2.3187 & 2005 & 2.1958 & 18 & 2.3009 & 2.1746 & 2.1690 & 1.9839 & 2.4357 & 2.3130 & 2.3093 & 2.1311 & 2.4485 & 2.3314 & 2.3281 & 2.1436 & 2.4362 & 2.3136 & 2.3098 & 2.1315 \\
\hline 6 & 2.3313 & 2.2070 & 2.2008 & 1.9859 & 2.3517 & 63 & 2.2 & & 2.3325 & 2.2084 & & 1.9867 & 66 & 2.3 & & & & & & & & 2.3463 & & 2.1343 \\
\hline 7 & 2.3574 & 2.2370 & 2.2282 & 1.9872 & 2.3793 & 2.2680 & 2.2605 & 2.0060 & 2.3588 & 2.2386 & 2.2297 & 1.9880 & 2.4923 & 2.3744 & 2.3683 & 2.1353 & 2.5071 & 2.3955 & 2.3902 & 2.1479 & 2.4930 & 2.3751 & 2.3691 & 2.1356 \\
\hline 8 & 2.3797 & 2.2589 & 2.2516 & 1.9878 & 2.4029 & 2919 & 2.2858 & & 2.3813 & 2.2606 & & 1.9887 & 2 & 2.3963 & & & & & & & & 2.3971 & 2.3921 & 2.1363 \\
\hline 9 & 2.3991 & 2.2797 & 2.2719 & 1.9881 & 2.4235 & 2.3144 & 2.3079 & 770 & 2.4008 & 2.2816 & 2.2737 & 1.9890 & 2.5335 & 2.4167 & 2.4113 & 2.1362 & 2.5501 & 2.4403 & 2.4357 & & 2.5344 & 2.4175 & 2.4122 & 2.1366 \\
\hline 10 & 2.4163 & 2.2981 & 2.2898 & 1.9883 & 2.4419 & & 2.3276 & & 2.41 & 2.3001 & & & & & & & & & & & 2.5514 & 2.4357 & 2.4300 & 2.1368 \\
\hline 11 & 2.4316 & 2.3146 & 2.3059 & 1.9884 & 2.4583 & 2.3523 & 2.3453 & 072 & 2.4336 & 2.3168 & 2.3081 & 1.9892 & 2.5658 & 2.4509 & 2.4450 & 2.1364 & & 2.4767 & 2.4716 & 2.1491 & 2.5668 & 2.4519 & 2.4460 & 2.1368 \\
\hline 12 & 2.4455 & 2.3295 & 2.3204 & 1.9884 & 2.4732 & 2.3686 & 2.3613 & 072 & 2.4476 & 2.3318 & 2.3227 & 1.9892 & 2.5796 & 2.4656 & 2.4594 & 2.1365 & 2.5984 & 2.4923 & 2.4870 & 2.1491 & 2.5806 & 2.4667 & 2.4605 & 2.1369 \\
\hline 13 & 2.4581 & 2.3431 & 2.3336 & 1.9884 & 2.4868 & 3835 & 2.3760 & & 2.4604 & 2.3455 & 2.3 & 1.9892 & 2 & 2.4 & 2.4 & 2.1365 & 2. & 2. & 2.5 & 2. & 2.5 & 2.4801 & 2.4737 & 2.1369 \\
\hline 14 & 2.4697 & 2.3555 & 2.3457 & 1.9884 & 2.4994 & 2.3972 & 2.3895 & & 2.47 & 2.3582 & & 1.9892 & 2.6037 & 2.4912 & 2.4 & & & & 2.5 & & 2.6050 & 2.4925 & 2.4858 & 2.1369 \\
\hline 15 & 2.4804 & 2.3670 & 2.3569 & & 2.5 & & & & & & & & & & & & & & & & & 2.5 & 2. & \\
\hline 16 & 2.4903 & 2.3777 & 2.3672 & 1.9884 & 2.5218 & 2.4218 & 2.4137 & & 2.4931 & 2.3807 & 2.3702 & 1.9892 & 2.6244 & 2.5132 & 2.5061 & 2.1365 & & & 2.5375 & & 2.6258 & 2.5147 & 2.5075 & 2.1369 \\
\hline 17 & 2.4995 & 2.3877 & 2.3769 & 1.9884 & 2.531 & 2.4329 & 2.4246 & & 2.5024 & 2.3908 & & & 2.63 & & 2.5 & 2.1365 & & & 2.5 & & 2.6351 & 2.5246 & 2.5173 & 2.1369 \\
\hline 18 & 2.5082 & 2.3970 & 2.3859 & 1.9884 & 2.5414 & 2.4434 & 2.4349 & 2.0073 & 2.5112 & 2.4003 & 2.3892 & 1.9892 & 2.6424 & 2.5324 & 2.5248 & 2.1365 & 2.6650 & 2.5642 & 2.5580 & 2.1491 & 2.6439 & 2.5340 & 2.5264 & 2.1369 \\
\hline 19 & 2.5163 & 2.4058 & 2.3944 & 1.9884 & 2.5503 & 2.4532 & 2.4446 & 2.0073 & 2519 & 2.409 & 2.3979 & 1.980 & 2.65 & 2.5 & & & 2.67 & 2.57 & & & 2.6521 & 2.5428 & 2.5350 & 2.1369 \\
\hline 20 & 2.5239 & 2.4140 & 2.4024 & 1.9884 & 2.5588 & 2.4625 & 2.4537 & 2.0073 & 2.5273 & 2.4176 & 2.4060 & 1.9892 & 2.6583 & 2.5493 & 2.5413 & 2.1365 & 2.6820 & 2.5826 & 2.5761 & 2.1491 & 2.6599 & 2.5511 & 2.5431 & 2.1369 \\
\hline
\end{tabular}


OOC $A R L$ comparison when $H=1,5$ and 10 with $\delta_{\max }=5$. For each $\delta \neq 0$, either in zero-state or steady-state, the charting scheme performing best is indicated in bold in the subsequent tables. If two or more values are boldfaced, then the charts perform similarly.

Table 4: The zero-state and steady-state OOC ARL values of the 2-of- $(H+1)$ synthetic and runsrules $\bar{X}$ charts when $H=1, \delta_{\max }=5$ and $A R L_{0}=370.4$

\begin{tabular}{|c|c|c|c|c|c|c|c|}
\hline \multirow[b]{3}{*}{$k$} & \multicolumn{4}{|c|}{$\begin{array}{c}\text { (a) } \\
\text { Zero-state }\end{array}$} & \multicolumn{2}{|r|}{$\begin{array}{c}\text { (b) } \\
\text { Steady-state }\end{array}$} & \multirow{2}{*}{$\begin{array}{l}\text { (c) } \\
\bar{X}\end{array}$} \\
\hline & DR & $\mathrm{KL}, \mathrm{MC} 1, \mathrm{AR}$ & WS & DW, MC2, MSS & DR, WS & KL, MC1, AR, DW, MC2, MSS & \\
\hline & 1.9323 & 1.7814 & 1.9435 & 1.7982 & 1.9328 & 1.7820 & 3.0000 \\
\hline 0 & 370.5 & 370.4 & 370.5 & 370.4 & 370.3 & 370.3 & 370.4 \\
\hline 0.1 & 354.83 & 342.00 & 354.27 & 341.14 & 354.70 & 341.92 & 352.93 \\
\hline 0.2 & 313.44 & 276.65 & 311.50 & 274.02 & 313.25 & 276.51 & 308.43 \\
\hline 0.3 & 258.94 & 207.12 & 255.41 & 203.06 & 258.69 & 206.93 & 253.14 \\
\hline 0.4 & 203.68 & 150.24 & 198.90 & 145.44 & 203.39 & 150.04 & 200.08 \\
\hline 0.5 & 155.29 & 108.45 & 149.77 & 103.42 & 154.98 & 108.24 & 155.22 \\
\hline 0.6 & 116.48 & 78.91 & 110.70 & 73.96 & 116.17 & 78.71 & 119.67 \\
\hline 0.7 & 86.93 & 58.21 & 81.24 & 53.51 & 86.63 & 58.02 & 92.32 \\
\hline 0.8 & 65.06 & 43.63 & 59.67 & 39.26 & 64.78 & 43.46 & 71.55 \\
\hline 0.9 & 49.09 & 33.25 & 44.10 & 29.23 & 48.83 & 33.10 & 55.83 \\
\hline 1 & 37.46 & 25.78 & 32.90 & 22.10 & 37.23 & 25.64 & 43.89 \\
\hline 1.1 & 28.97 & 20.32 & 24.84 & 16.96 & 28.76 & 20.19 & 34.80 \\
\hline 1.2 & 22.74 & 16.28 & 19.00 & 13.21 & 22.55 & 16.16 & 27.82 \\
\hline 1.3 & 18.11 & 13.24 & 14.73 & 10.45 & 17.94 & 13.14 & 22.43 \\
\hline 1.4 & 14.64 & 10.94 & 11.58 & 8.38 & 14.49 & 10.84 & 18.25 \\
\hline 1.5 & 12.02 & 9.17 & 9.24 & 6.82 & 11.88 & 9.08 & 14.97 \\
\hline 1.6 & 10.01 & 7.79 & 7.47 & 5.63 & 9.88 & 7.71 & 12.38 \\
\hline 1.7 & 8.45 & 6.71 & 6.13 & 4.71 & 8.33 & 6.64 & 10.33 \\
\hline 1.8 & 7.23 & 5.85 & 5.09 & 3.99 & 7.12 & 5.78 & 8.69 \\
\hline 1.9 & 6.27 & 5.17 & 4.29 & 3.42 & 6.17 & 5.10 & 7.37 \\
\hline 2 & 5.50 & 4.61 & 3.66 & 2.97 & 5.41 & 4.55 & 6.30 \\
\hline 2.1 & 4.88 & 4.16 & 3.16 & 2.61 & 4.79 & 4.10 & 5.43 \\
\hline 2.2 & 4.38 & 3.79 & 2.77 & 2.32 & 4.30 & 3.73 & 4.72 \\
\hline 2.3 & 3.97 & 3.49 & 2.45 & 2.09 & 3.89 & 3.43 & 4.13 \\
\hline 2.4 & 3.63 & 3.23 & 2.19 & 1.90 & 3.56 & 3.18 & 3.65 \\
\hline 2.5 & 3.36 & 3.02 & 1.98 & 1.74 & 3.29 & 2.97 & 3.24 \\
\hline 2.6 & 3.13 & 2.85 & 1.81 & 1.61 & 3.06 & 2.80 & 2.90 \\
\hline 2.7 & 2.93 & 2.70 & 1.66 & 1.50 & 2.87 & 2.66 & 2.62 \\
\hline 2.8 & 2.77 & 2.58 & 1.55 & 1.41 & 2.71 & 2.54 & 2.38 \\
\hline 2.9 & 2.64 & 2.48 & 1.45 & 1.34 & 2.58 & 2.43 & 2.17 \\
\hline 3 & 2.53 & 2.39 & 1.37 & 1.28 & 2.47 & 2.35 & 2.00 \\
\hline 3.1 & 2.43 & 2.32 & 1.30 & 1.23 & 2.38 & 2.28 & 1.85 \\
\hline 3.2 & 2.36 & 2.26 & 1.25 & 1.18 & 2.30 & 2.22 & 1.73 \\
\hline 3.3 & 2.29 & 2.21 & 1.20 & 1.15 & 2.24 & 2.17 & 1.62 \\
\hline 3.4 & 2.24 & 2.17 & 1.16 & 1.12 & 2.18 & 2.13 & 1.53 \\
\hline 3.5 & 2.19 & 2.14 & 1.13 & 1.10 & 2.14 & 2.10 & 1.45 \\
\hline 3.6 & 2.15 & 2.11 & 1.11 & 1.08 & 2.10 & 2.07 & 1.38 \\
\hline 3.7 & 2.12 & 2.09 & 1.08 & 1.06 & 2.07 & 2.05 & 1.32 \\
\hline 3.8 & 2.10 & 2.07 & 1.07 & 1.05 & 2.04 & 2.03 & 1.27 \\
\hline 3.9 & 2.08 & 2.05 & 1.05 & 1.04 & 2.02 & 2.01 & 1.23 \\
\hline 4 & 2.06 & 2.04 & 1.04 & 1.03 & 2.01 & 2.00 & 1.19 \\
\hline 4.1 & 2.05 & 2.03 & 1.03 & 1.02 & 1.99 & 1.99 & 1.16 \\
\hline 4.2 & 2.04 & 2.02 & 1.02 & 1.02 & 1.98 & 1.98 & 1.13 \\
\hline 4.3 & 2.03 & 2.02 & 1.02 & 1.01 & 1.98 & 1.98 & 1.11 \\
\hline 4.4 & 2.02 & 2.01 & 1.01 & 1.01 & 1.97 & 1.97 & 1.09 \\
\hline 4.5 & 2.02 & 2.01 & 1.01 & 1.01 & 1.96 & 1.97 & 1.07 \\
\hline 4.6 & 2.01 & 2.01 & 1.01 & 1.01 & 1.96 & 1.97 & 1.06 \\
\hline 4.7 & 2.01 & 2.01 & 1.01 & 1.00 & 1.96 & 1.97 & 1.05 \\
\hline 4.8 & 2.01 & 2.00 & 1.00 & 1.00 & 1.96 & 1.97 & 1.04 \\
\hline 4.9 & 2.00 & 2.00 & 1.00 & 1.00 & 1.95 & 1.96 & 1.03 \\
\hline 5 & 2.00 & 2.00 & 1.00 & 1.00 & 1.95 & 1.96 & 1.02 \\
\hline EQL & 299.43 & 262.45 & 196.66 & 163.75 & 294.23 & 258.63 & 253.99 \\
\hline ARARL & 1.8330 & 1.6257 & 1.1790 & 1.0000 & 1.1558 & 1.0000 & \\
\hline PCI & 1.8286 & 1.6027 & 1.2010 & 1.0000 & 1.1377 & 1.0000 & \\
\hline
\end{tabular}


Table 5: The zero-state and steady-state OOC $A R L$ values of the 2-of- $(H+1)$ synthetic and runsrules $\bar{X}$ charts when $H=5, \delta_{\max }=5$ and $A R L_{0}=370.4$

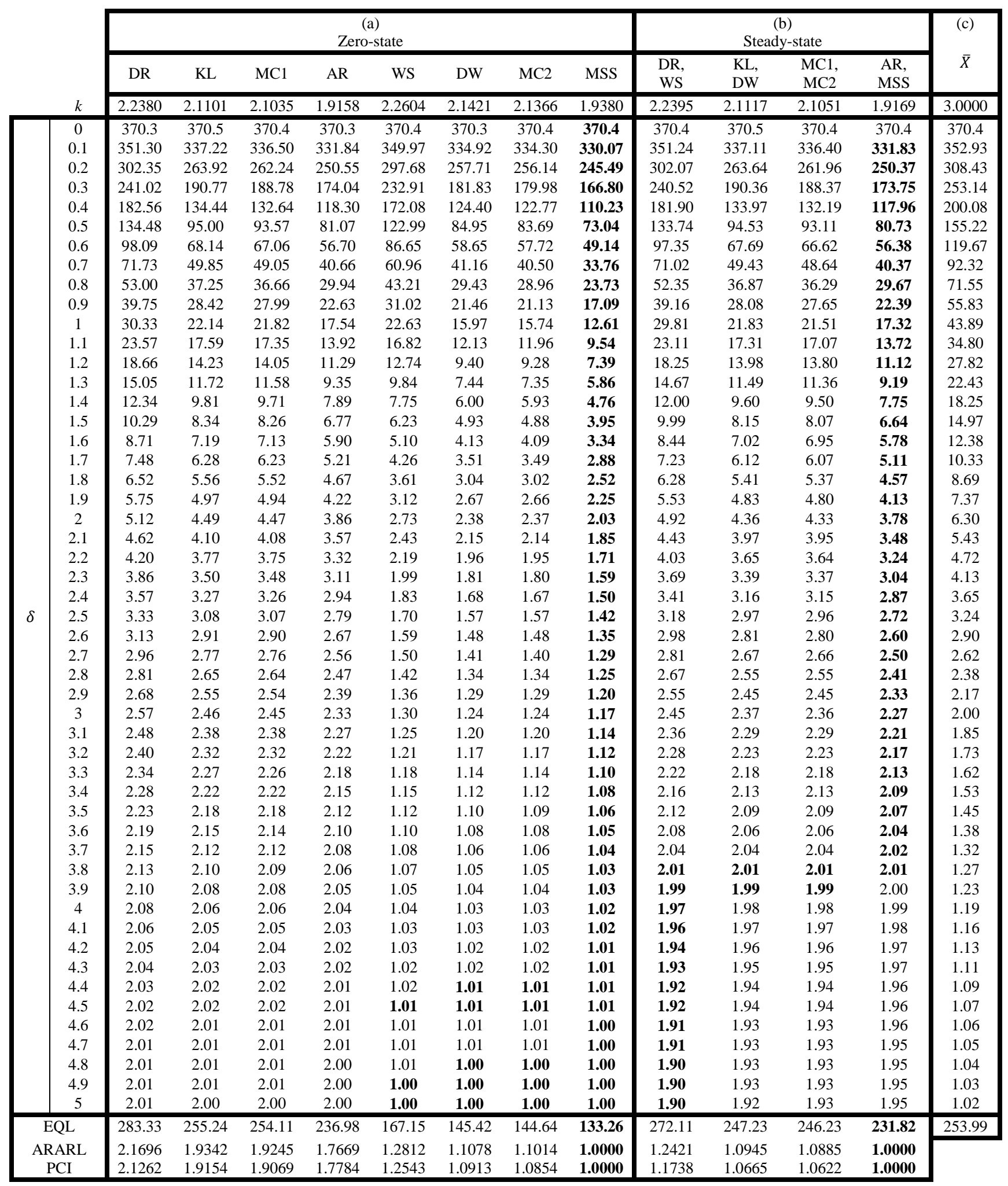


Table 6: The zero-state and steady-state OOC $A R L$ values of the 2-of- $(H+1)$ synthetic and runsrules $\bar{X}$ charts when $H=10, \delta_{\max }=5$ and $A R L_{0}=370.4$

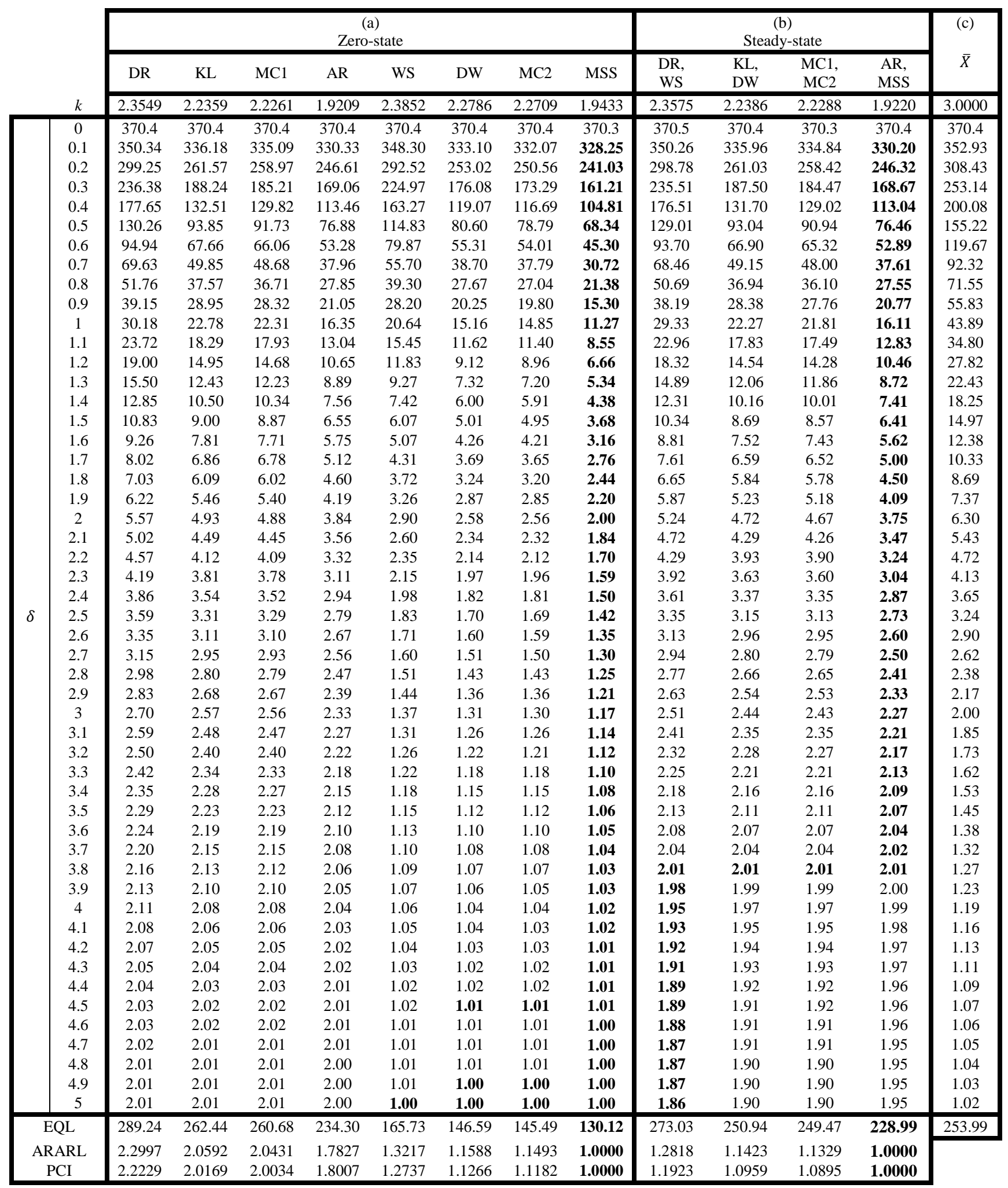


Remark 1. Since the MSS, MC2 and DW schemes are all side-sensitive, the only difference is the manner in which the $C R L$ values are computed, see Sections 1 and 2.1 . When $H=1$ there are $(H-1=)$ zero conforming samples in between the two consecutive nonconforming samples which implies that the $C R L$ is equal to 1 (i.e. the nonconforming sample at the end) for the three schemes. Thus, it follows that when $H=1$, in zero-state, the OOC performance of the DW, MC2 and MSS charts are equivalent i.e. $\mathrm{MSS} \equiv \mathrm{MC} 2 \equiv \mathrm{DW}$ and also in steady-state, $\mathrm{MSS} \equiv \mathrm{MC} 2 \equiv \mathrm{DW}$; see also their state spaces in Table 2 for further proof. Similarly, using the same argument, it follows that when $H=1$, $\mathrm{AR} \equiv \mathrm{MC1} \equiv \mathrm{KL}$. Furthermore, in Section 2.2 we showed that when we remove the elements with a HS feature on the TPMs of MSS, MC2 and DW, these reduce to the TPMs of the AR, MC1 and $\mathrm{KL}$, respectively, see Equations (5) to (7); hence in steady-state, $\mathrm{MSS} \equiv \mathrm{AR}, \mathrm{MC} 2 \equiv \mathrm{MC} 1$ and $\mathrm{DW} \equiv \mathrm{KL}$ for all $H>0$. Moreover, Davis and Woodall [10] showed that in steady-state, WS $\equiv \mathrm{DR}$ for all $H>0$ (we also showed that the TPMs of WS and DR are the same, see Equation (4)). Therefore based on this, we conclude that when $H=1, \mathrm{MSS} \equiv \mathrm{MC} 2 \equiv \mathrm{DW}$ and $\mathrm{AR} \equiv \mathrm{MC} 1 \equiv \mathrm{KL}$ in zero-state, whereas $\mathrm{MSS} \equiv \mathrm{MC} 2 \equiv \mathrm{DW} \equiv \mathrm{AR} \equiv \mathrm{MC} 1 \equiv \mathrm{KL}$ and $\mathrm{WS} \equiv \mathrm{DR}$ in steady-state.

Remark 2. In zero-state, the OOC ARLs of the DR, KL, MC1, AR schemes converges to 2 whereas those of the WS, DW, MC2 and MSS converge to 1; see Panel (a) of Tables 4 to 6. This convergence is to be expected because the main drawback of the DR, KL, MC1 and AR schemes (in both zero-state and steady-state) is the lack of being able to immediately detect a large process shift; that is, it requires at least two plotting statistics to issue a signal. This drawback only occurs in steady-state for the WS, DW, MC2 and MSS schemes; thus in Panel (b) of Tables 4 to 6, the OOC $A R L$ s converge to approximately 2, whereas in Panel (a) the OOC ARLs converge to 1 because of the HS feature assumption.

In Panel (a) of Table 4, the ZSARL of the MSS, MC2 and DW are uniformly better than all the schemes in Table 1 and hence have the lowest EQL. In Panel (b) the six schemes (MSS, MC2, DW, AR, MC1, KL) have uniformly better $S S A R L$ than DR and WS when $\delta \leq 4.4$, however, when $\delta$ $>4.4$ the DR and WS outperform the other six schemes. Moreover, the $\bar{X}$ chart has better OOC $A R L$ 
performance than DR, KL, AR (in zero-state) and all schemes in steady-state when $\delta \geq 2.7$, hence for these schemes the $\bar{X}$ chart has the smallest $E Q L$. Note that in Table 5 and 6, Remark 1 does not hold anymore, so that only the MSS scheme has a uniformly better ZSARL than all the schemes.

The results in these three tables are further discussed below in Figures 2 to 4. In Panel (c) of Tables 4 to 6 we added the OOC $A R L$ of the $\bar{X}$ chart and computed the $E Q L$ as 253.99 . Note that for $\delta_{\max }=$ 5 the $E Q L$ values of the $\bar{X}$ chart are equal to $196.31,292.43$ and 420.35 when $A R L_{0}=200,500$ and 1000 , respectively. Similarly, for $\delta_{\max }=3$ the $E Q L$ values are equal to $199.50,289.09,349.33$ and 551.79 and finally, for $\delta_{\max }=2$ these are equal to $216.28,331.87,410.94$ and 681.57 when $A R L_{0}=$ 200, 370.4, 500 and 1000, respectively. These $E Q L$ values for the $\bar{X}$ chart are overlaid as reference values in Figures 2 to 4 to make overall performance comparison with the charts listed in Table 1.

Table 7: The zero-state and steady-state $E Q L$ values of the 2-of- $(H+1)$ synthetic and runs-rules $\bar{X}$ charts when $H=1,2, \ldots, 20$ for $\delta_{\max }=5$ and $A R L_{0}=370.4$

\begin{tabular}{|c|c|c|c|c|c|c|c|c|c|c|c|c|}
\hline \multirow[b]{2}{*}{$H$} & \multicolumn{8}{|c|}{ Zero-state } & \multicolumn{4}{|c|}{ Steady-state } \\
\hline & DR & KL & $\mathrm{MC1}$ & $\mathrm{AR}$ & WS & DW & $\mathrm{MC} 2$ & MSS & DR \& WS & KL \& DW & MC1 \& MC2 & AR \& MSS \\
\hline 1 & 299.43 & 262.45 & 262.45 & 262.45 & 196.66 & 163.75 & 163.75 & 163.75 & 294.23 & 258.63 & 258.63 & 258.63 \\
\hline 2 & 285.98 & 254.13 & 253.65 & 247.37 & 178.03 & 151.18 & 150.77 & 145.85 & 278.96 & 249.05 & 248.60 & 242.86 \\
\hline 3 & 283.09 & 253.25 & 252.50 & 241.72 & 171.71 & 147.44 & 146.86 & 138.97 & 274.52 & 247.08 & 246.38 & 236.86 \\
\hline 4 & 282.77 & 254.00 & 253.02 & 238.74 & 168.74 & 145.96 & 145.27 & 135.36 & 272.77 & 246.83 & 245.98 & 233.68 \\
\hline 5 & 283.33 & 255.24 & 254.11 & 236.98 & 167.15 & 145.42 & 144.64 & 133.26 & 272.11 & 247.23 & 246.23 & 231.82 \\
\hline 6 & 284.32 & 256.66 & 255.38 & 235.90 & 166.29 & 145.34 & 144.47 & 131.97 & 271.96 & 247.85 & 246.73 & 230.67 \\
\hline 7 & 285.46 & 258.53 & 256.71 & 235.21 & 165.83 & 145.75 & 144.55 & 131.16 & 272.04 & 248.88 & 247.38 & 229.94 \\
\hline 8 & 286.71 & 259.59 & 258.06 & 234.77 & 165.66 & 145.78 & 144.77 & 130.66 & 272.29 & 249.37 & 248.07 & 229.47 \\
\hline 9 & 287.96 & 261.04 & 259.39 & 234.49 & 165.64 & 146.16 & 145.11 & 130.33 & 272.63 & 250.17 & 248.77 & 229.18 \\
\hline 10 & 289.24 & 262.44 & 260.68 & 234.30 & 165.73 & 146.59 & 145.49 & 130.12 & 273.03 & 250.94 & 249.47 & 228.99 \\
\hline 11 & 290.47 & 263.78 & 261.93 & 234.19 & 165.90 & 147.07 & 145.92 & 129.99 & 273.42 & 251.72 & 250.17 & 228.87 \\
\hline 12 & 291.72 & 265.10 & 263.12 & 234.11 & 166.15 & 147.57 & 146.37 & 129.91 & 273.83 & 252.47 & 250.87 & 228.79 \\
\hline 13 & 292.89 & 266.36 & 264.29 & 234.06 & 166.44 & 148.07 & 146.84 & 129.85 & 274.26 & 253.19 & 251.53 & 228.74 \\
\hline 14 & 294.08 & 267.58 & 265.42 & 234.03 & 166.74 & 148.59 & 147.31 & 129.82 & 274.71 & 253.90 & 252.18 & 228.71 \\
\hline 15 & 295.21 & 268.76 & 266.50 & 234.01 & 167.09 & 149.12 & 147.80 & 129.79 & 275.12 & 254.57 & 252.79 & 228.68 \\
\hline 16 & 296.33 & 269.90 & 267.57 & 234.00 & 167.44 & 149.63 & 148.28 & 129.78 & 275.53 & 255.23 & 253.39 & 228.67 \\
\hline 17 & 297.38 & 271.01 & 268.58 & 233.99 & 167.81 & 150.15 & 148.76 & 129.77 & 275.95 & 255.87 & 253.99 & 228.66 \\
\hline 18 & 298.44 & 272.07 & 269.55 & 233.98 & 168.17 & 150.67 & 149.24 & 129.76 & 276.36 & 256.49 & 254.54 & 228.66 \\
\hline 19 & 299.47 & 273.10 & 270.50 & 233.98 & 168.55 & 151.19 & 149.74 & 129.76 & 276.74 & 257.19 & 255.11 & 228.65 \\
\hline 20 & 300.44 & 274.12 & 271.42 & 233.98 & 168.94 & 151.69 & 150.20 & 129.75 & 277.14 & 257.66 & 255.63 & 228.65 \\
\hline
\end{tabular}

In Table 7, we use Equation (13) to compute the zero-state and steady-state EQL values when the mean shifts from 0 to 5 in step size of 0.1 and $A R L_{0}=370.4$. We see that in zero-state, the MSS chart results in a uniformly better overall performance (except when $H=1$, see Remark 1) compared to the other competing schemes as $H$ varies from 1 to 20 . Similarly, in steady-state, the MSS (and AR) have a uniformly better overall performance than the competing charts (except when 

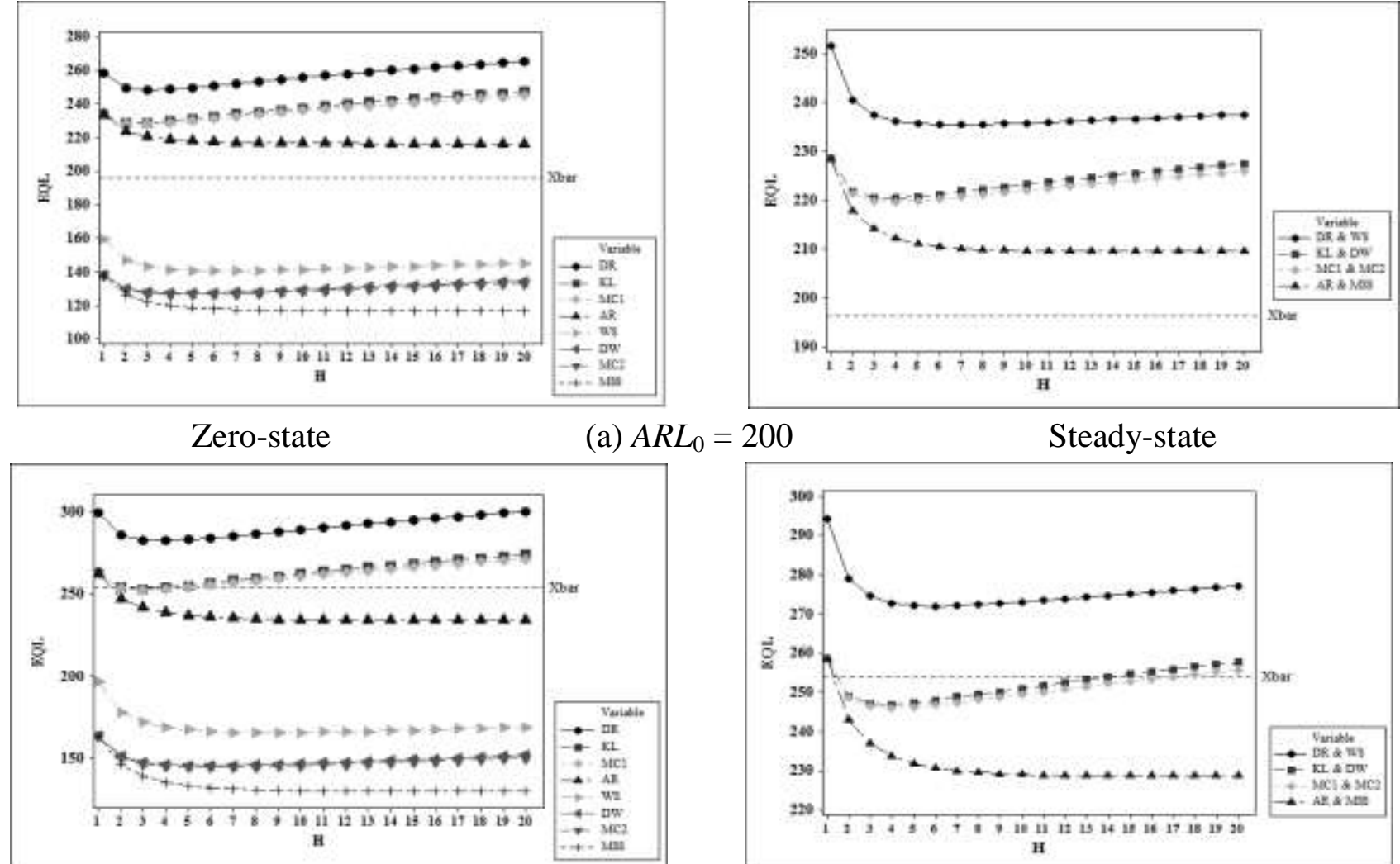

(a) $A R L_{0}=200$

Steady-state
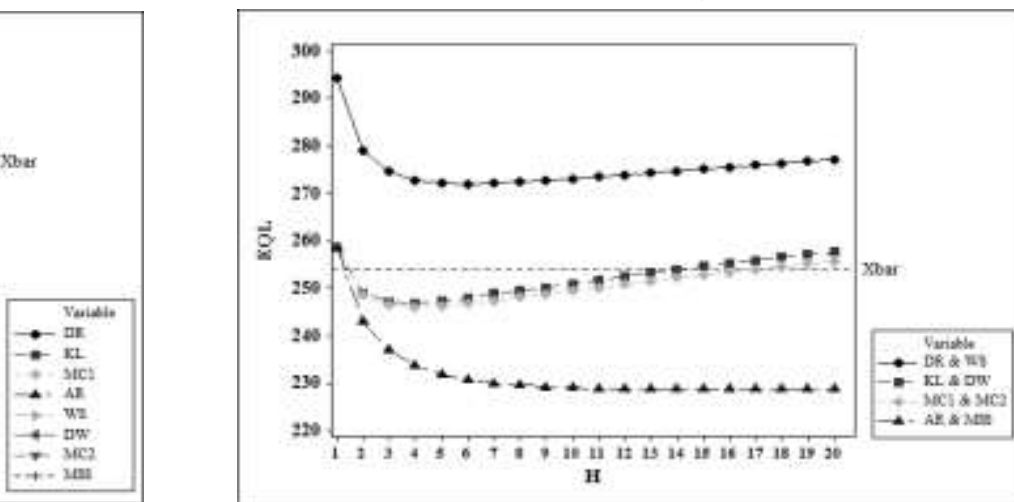

\section{Zero-state}

(b) $A R L_{0}=370.4$

Steady-state
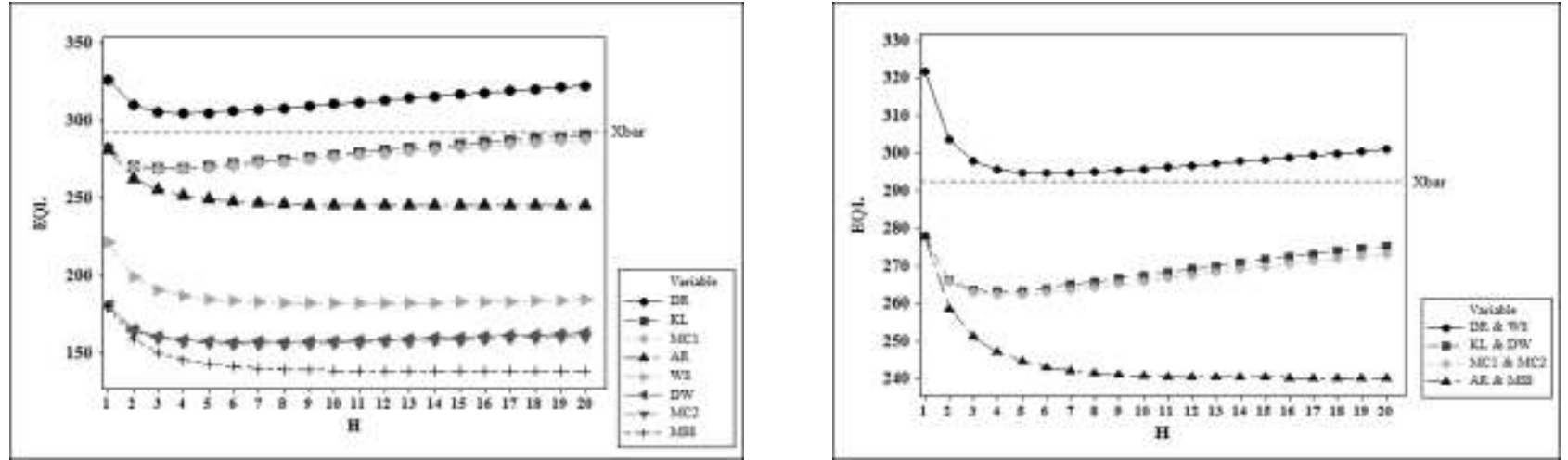

Zero-state

(c) $A R L_{0}=500$

Steady-state
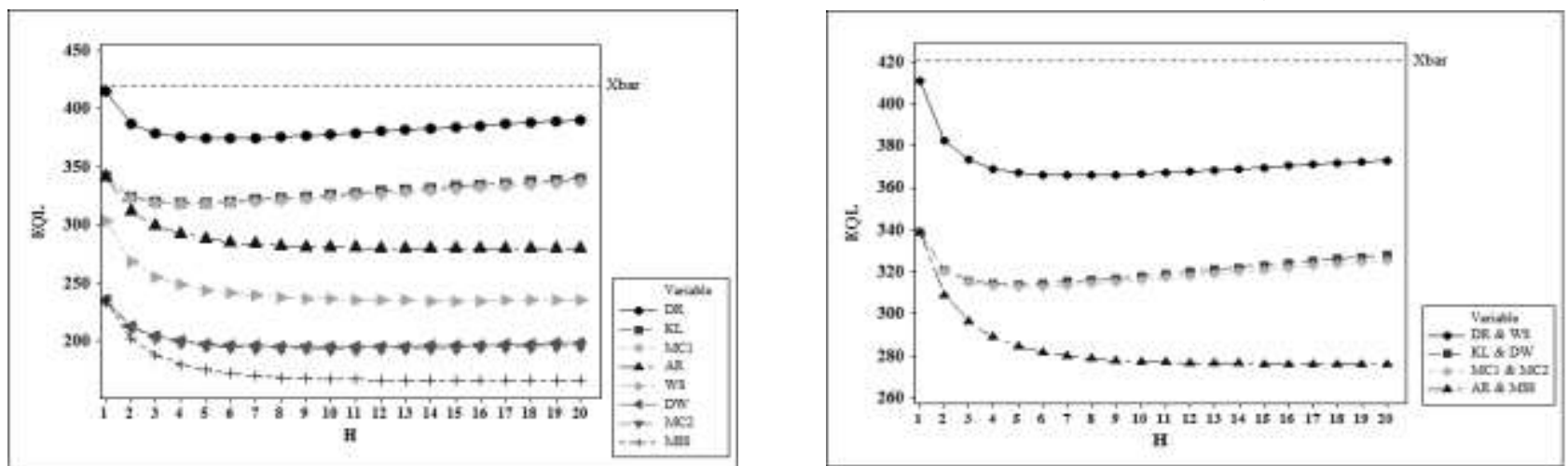

Zero-state

(d) $A R L_{0}=1000$

Steady-state

Figure 2: The zero-state and steady-state $E Q L$ values of the 2-of- $(H+1)$ synthetic and runs-rules $\bar{X}$ charts when $H=1,2, \ldots, 20$ for $\delta_{\max }=5$ and $A R L_{0}=200,370.4,500,1000$ 

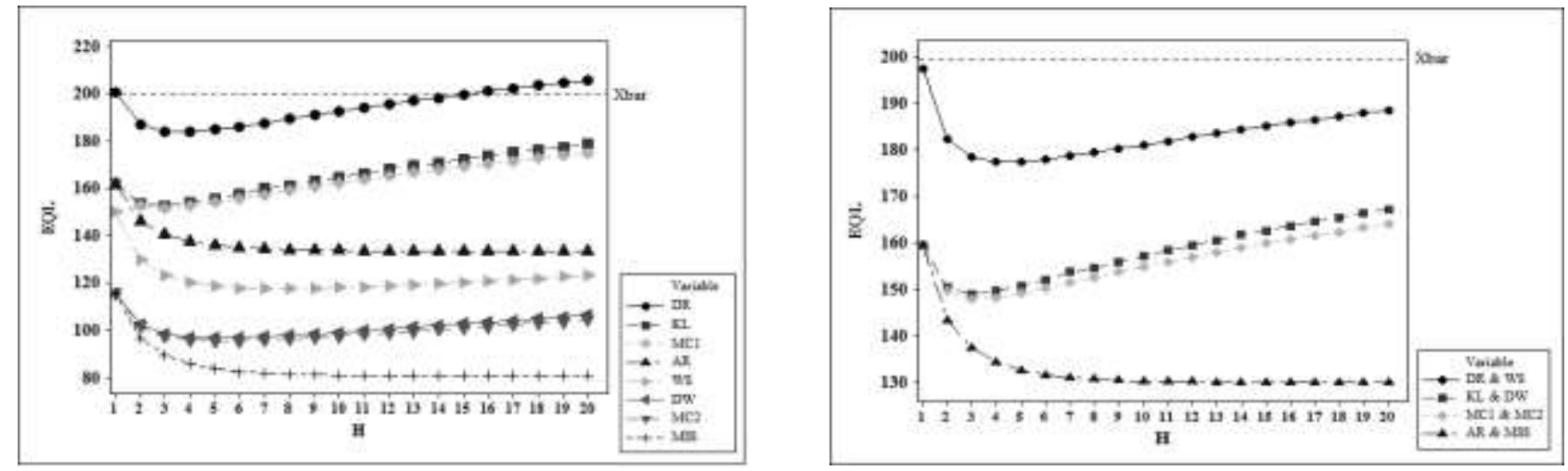

Zero-state

(a) $A R L_{0}=200$

Steady-state
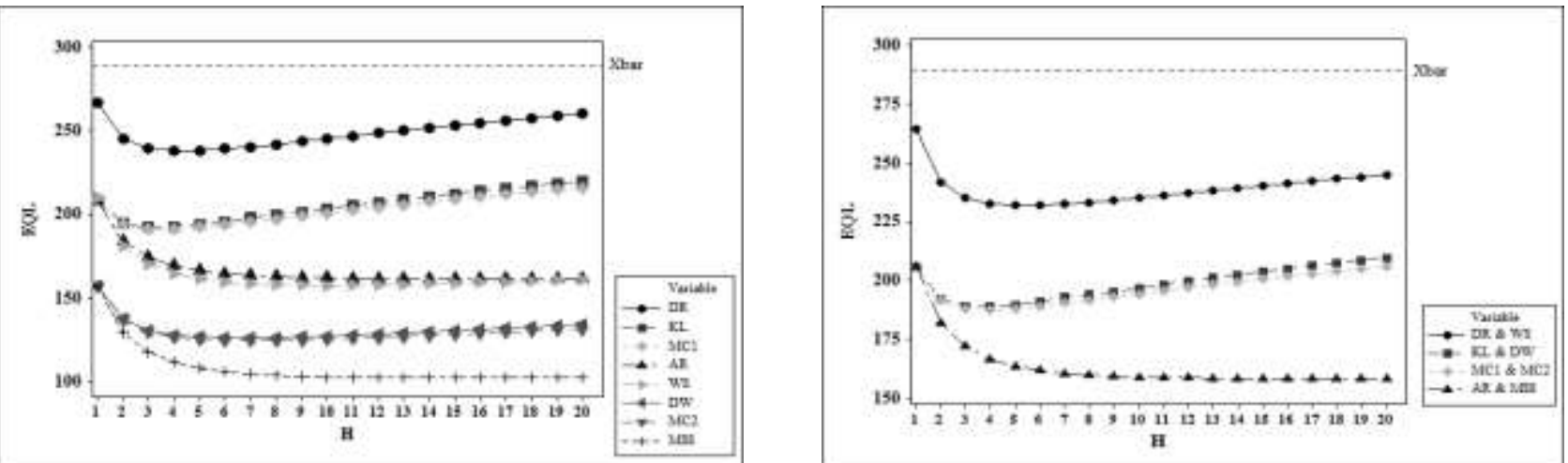

\section{Zero-state}

(b) $A R L_{0}=370.4$

Steady-state
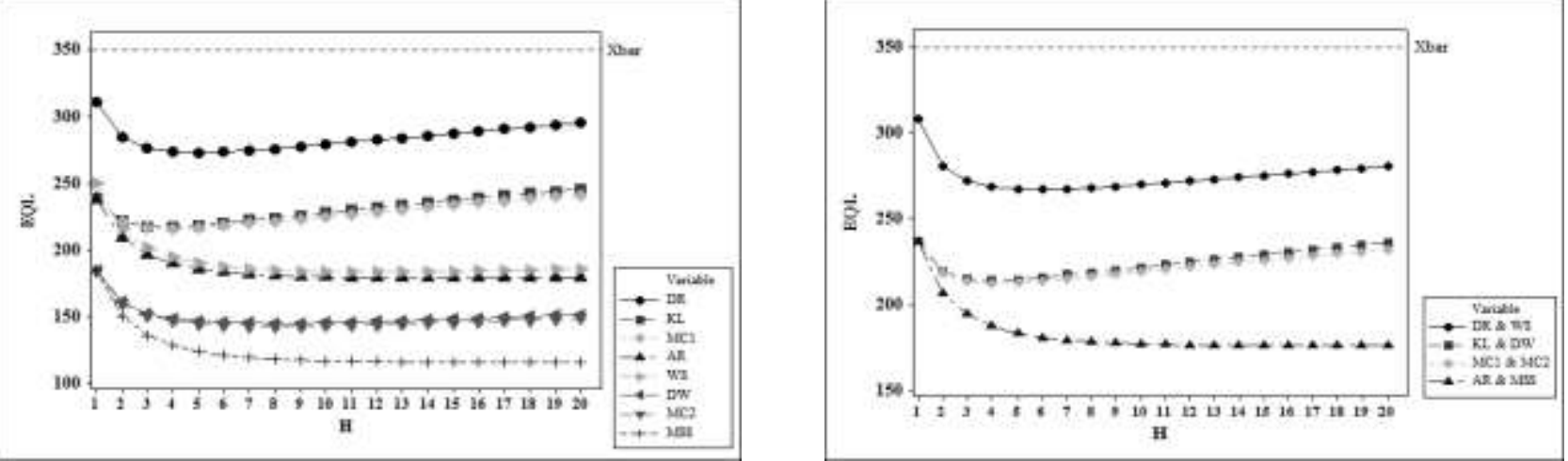

Zero-state

(c) $A R L_{0}=500$

Steady-state
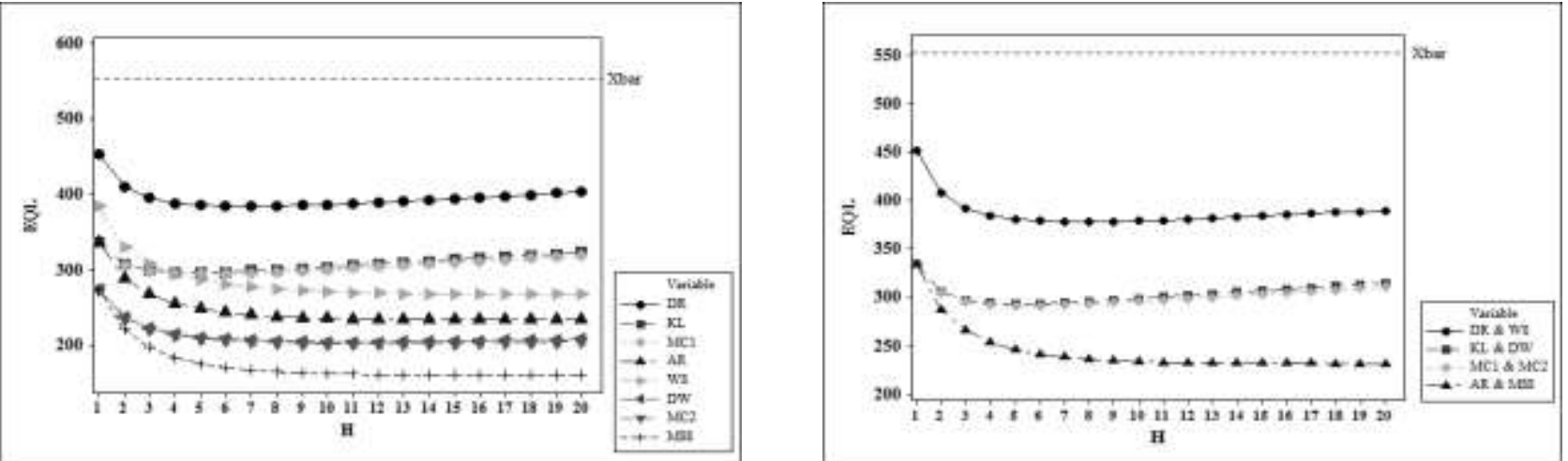

Zero-state

(d) $A R L_{0}=1000$

Steady-state

Figure 3: The zero-state and steady-state $E Q L$ values of the 2-of-(H+1) synthetic and runs-rules $\bar{X}$ charts when $H=1,2, \ldots, 20$ for $\delta_{\max }=3$ and $A R L_{0}=200,370.4,500,1000$ 


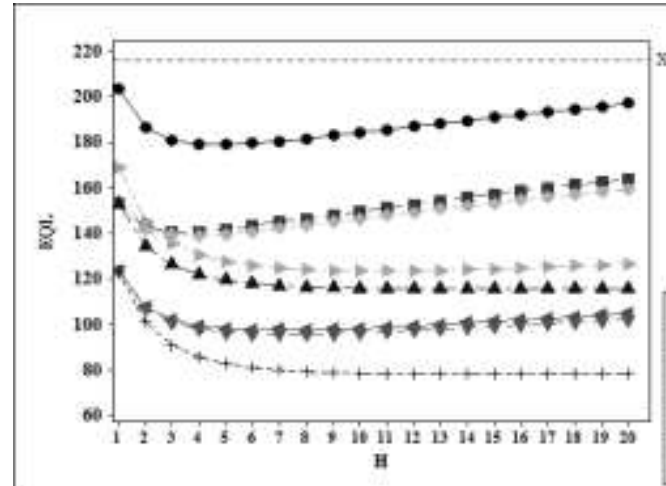

Zero-state

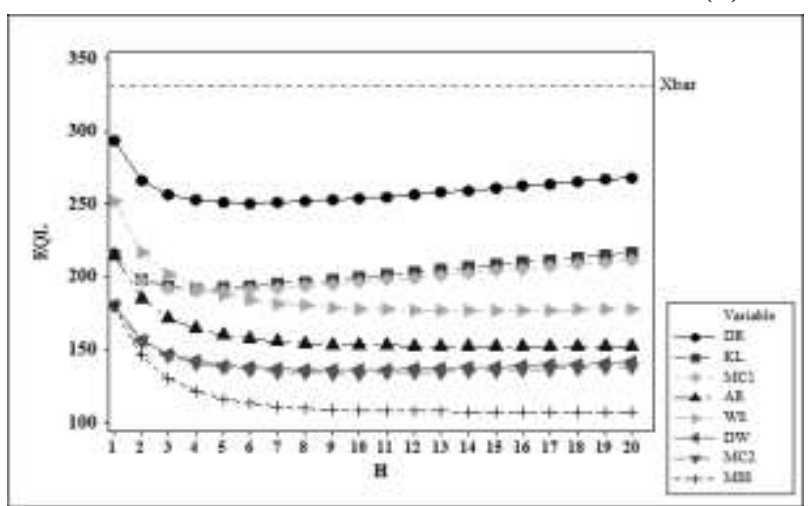

Zero-state

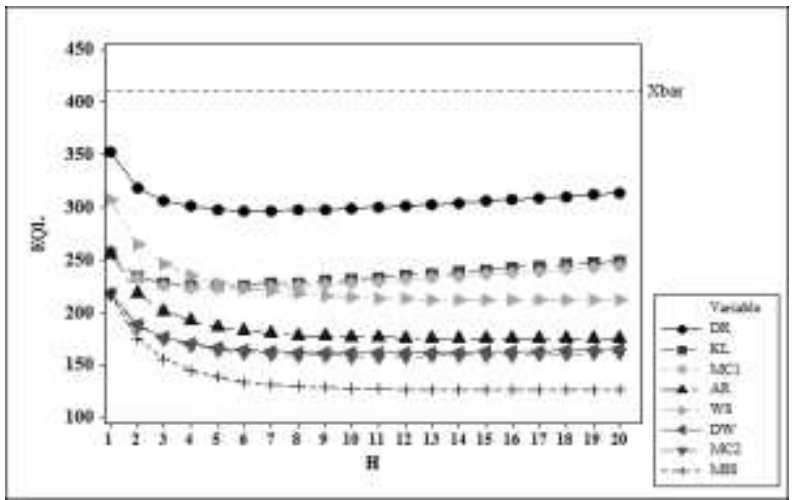

Zero-state

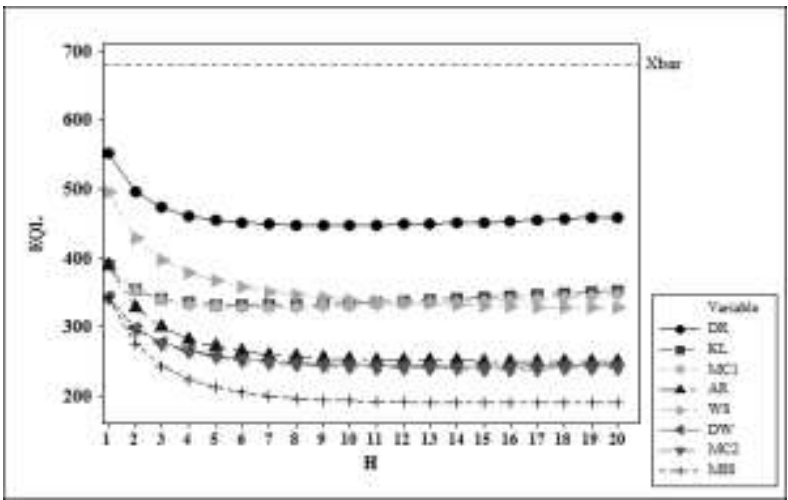

Zero-state

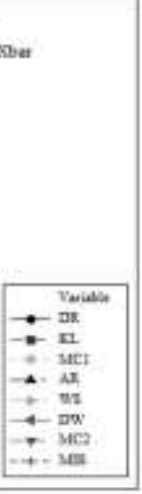

(a) $A R L_{0}=200$

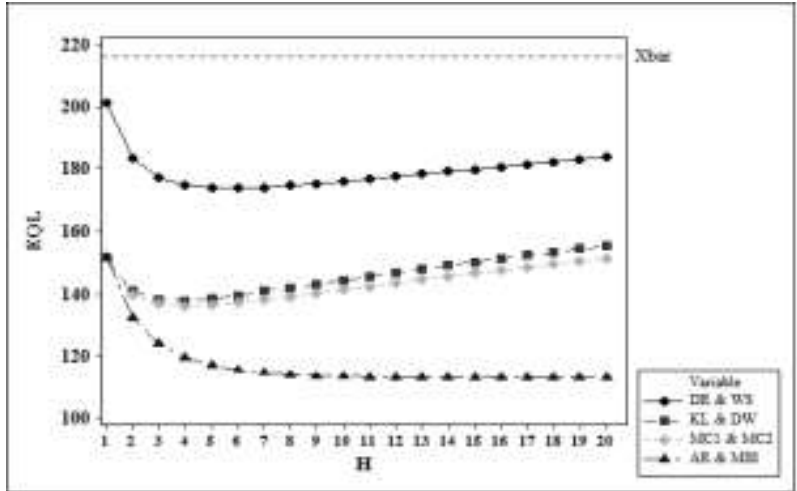

Steady-state

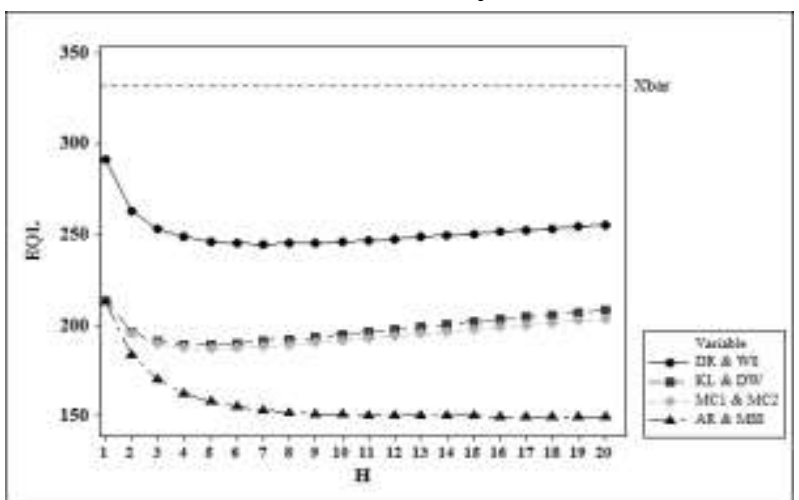

(b) $A R L_{0}=370.4$

Steady-state

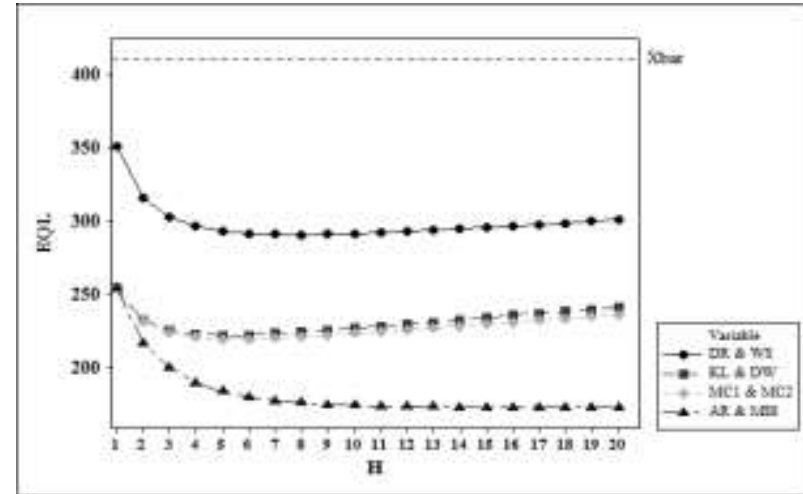

(c) $A R L_{0}=500$

Steady-state

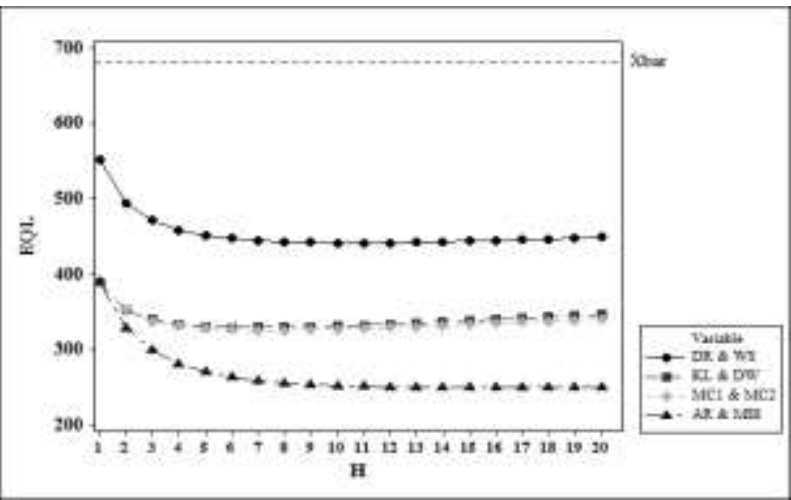

(d) $A R L_{0}=1000$

Steady-state

Figure 4: The zero-state and steady-state $E Q L$ values of the 2-of- $(H+1)$ synthetic and runs-rules $\bar{X}$ charts when $H=1,2, \ldots, 20$ for $\delta_{\max }=2$ and $A R L_{0}=200,370.4,500,1000$ 
$H=1$, see Remark 1). To better illustrate the behavior of the $E Q L$ values in Table 7, these are shown in Figure 2, Panel (b). In addition, we graphically illustrate the $E Q L$ values for other values of $A R L_{0}$ (i.e. $200,370.4,500,1000$ ) in Figures 2,3 and 4 where $\delta_{\max }=5,3$ and 2, respectively.

In Figures 2 to 4 , we see that for a variety of $A R L_{0}, \delta_{\max }$ and $H$ values, the MSS chart has a uniformly lower $E Q L$ values than the other schemes listed in Table 1 for the zero-state and steadystate, respectively. Except in two instances in steady-state (see Figures 2 (a) and when $H=1$ in Figure 2(b)), the MSS chart always has a much larger improvement on the $\bar{X}$ chart than the other schemes. We see that the DW and MC2, as well as KL and MC1, have an approximately equal overall performance, however, the MC2 and MC1 schemes have a relatively lower $E Q L$ values. Moreover, as $\delta_{\max }$ (for each $A R L_{0}$ ) decrease, the synthetic and runs-rules charts tend to have a better performance than the $\bar{X}$ chart; also the runs-rules schemes become more competitive and in some cases perform better than synthetic chart, see for instance Figure 4(d). As $A R L_{0}$ (for each $\delta_{\max }$ ) increase, the synthetic and runs-rules charts tend to have a better performance than the $\bar{X}$ chart and the AR scheme becomes more competitive in zero-state. The convergence in the optimal values of $k$ shown in Table 3, leads to convergence in the overall performance of the MSS and AR schemes. However, the overall performance of DR, KL, MC1, WS, DW and MC2 seem to decrease and then at some specific value of $H$, the $E Q L$ increases; this phenomenon is more evident in the steady-state mode. Thus, in Table 8, we recommend the values of $H$ where the $E Q L$ is the smallest (i.e. the minimum of the curves in Figures 2 to 4 ), as the 'optimal' $H$ (denoted by $H^{*}$ ) to use in the 2-of$(H+1)$ charts, respectively. Consider Table 7, we see that the EQL values of the MSS scheme converge to approximately 129 , but, at a slow rate. Hence, for the MSS and AR schemes, we recommend the values of $H^{*}$ such that $\left|\frac{E Q L_{H^{*}}-E Q L_{H=20}}{E Q L_{H=20}}\right|<0.01$; this means that the percentage improvement (if any) in $E Q L$ value for any value greater or equal to $H^{*}$ is only less than $1 \%$ compared to the smallest $E Q L$ value at $H=20$. For example, from Table 7 where $A R L_{0}=370.4$ and 
$\delta_{\max }=5$, the value of $H^{*}$ is $\geq 6$ and $\geq 8$ for the AR and MSS schemes in zero-state, respectively; whereas in steady-state $H^{*} \geq 6$, see Table 8 .

Table 8: The values of $H^{*}$ for the synthetic and runs-rules $\bar{X}$ charts when $\delta_{\max }=2,3,5$ and $A R L_{0}=$ $200,370.4,500,1000$ such that $E Q L$ is (approximately) minimum

\begin{tabular}{|c|c|c|c|c|c|c|c|c|c|c|c|c|c|}
\hline \multirow[b]{2}{*}{$A R L_{0}$} & \multirow[b]{2}{*}{$\delta_{\max }$} & \multicolumn{8}{|c|}{ Zero-state } & \multicolumn{4}{|c|}{ Steady-state } \\
\hline & & DR & KL & MC1 & $\mathrm{AR}$ & WS & DW & $\mathrm{MC} 2$ & MSS & $\begin{array}{c}\text { DR \& } \\
\text { WS }\end{array}$ & $\begin{array}{c}\text { KL \& } \\
\text { DW }\end{array}$ & $\begin{array}{l}\text { MC1 \& } \\
\text { MC2 }\end{array}$ & $\begin{array}{c}\text { AR \& } \\
\text { MSS }\end{array}$ \\
\hline \multirow{3}{*}{200} & 2 & 5 & 3 & 4 & $\geq 8$ & 10 & 6 & 7 & $\geq 9$ & 6 & 4 & 4 & $\geq 8$ \\
\hline & 3 & 4 & 3 & 3 & $\geq 7$ & 7 & 5 & 5 & $\geq 8$ & 5 & 3 & 3 & $\geq 7$ \\
\hline & 5 & 3 & 2 & 3 & $\geq 5$ & 6 & 4 & 5 & $\geq 6$ & 7 & 4 & 4 & $\geq 5$ \\
\hline \multirow{3}{*}{370.4} & 2 & 6 & 4 & 5 & $\geq 9$ & 14 & 9 & 10 & $\geq 10$ & 7 & 5 & 5 & $\geq 9$ \\
\hline & 3 & 4 & 3 & 3 & $\geq 8$ & 10 & 6 & 7 & $\geq 9$ & 5 & 4 & 4 & $\geq 8$ \\
\hline & 5 & 4 & 3 & 3 & $\geq 6$ & 9 & 6 & 6 & $\geq 8$ & 6 & 4 & 4 & $\geq 6$ \\
\hline \multirow{3}{*}{500} & 2 & 7 & 5 & 5 & $\geq 10$ & 16 & 10 & 11 & $\geq 11$ & 8 & 5 & 6 & $\geq 10$ \\
\hline & 3 & 5 & 4 & 4 & $\geq 9$ & 12 & 8 & 8 & $\geq 10$ & 6 & 4 & 4 & $\geq 9$ \\
\hline & 5 & 4 & 3 & 3 & $\geq 7$ & 10 & 6 & 7 & $\geq 8$ & 6 & 4 & 4 & $\geq 7$ \\
\hline \multirow{3}{*}{1000} & 2 & 9 & 6 & 7 & $\geq 11$ & 20 & 15 & 16 & $\geq 12$ & 10 & 6 & 7 & $\geq 11$ \\
\hline & 3 & 7 & 5 & 5 & $\geq 10$ & 17 & 11 & 12 & $\geq 11$ & 8 & 5 & 5 & $\geq 10$ \\
\hline & 5 & 6 & 4 & 4 & $\geq 8$ & 14 & 9 & 10 & $\geq 10$ & 7 & 5 & 5 & $\geq 8$ \\
\hline
\end{tabular}

Furthermore, in Table 9 it can be seen that the values of the relative effectiveness of the MSS scheme computed using Equation (14) and the EQL values in Table 7 yield PCI values that are greater than 1 for all the competing schemes listed in Table 1 (except when $H=1$, see Remark 1). For instance, in zero-state, from the overall viewpoint, the MSS scheme yields a performance that is more than $100 \%$ better than that of DR and KL schemes, i.e. as $H$ increases from 1 to 20 , the quality loss in using the DR chart varies from $82.86 \%$ to $131.55 \%$, whereas for the KL scheme it varies from $60.27 \%$ to $111.26 \%$. Similarly, the quality loss in using the MC1, AR, WS, DW and MC2 schemes ranges from $60.27 \%, 60.27 \%, 20.10 \%, 0 \%$ and $0 \%$ to $109.18 \%, 80.32 \%, 30.20 \%, 16.91 \%$ and $15.76 \%$, respectively. However, in steady-state the quality loss improvement is not as significant as it is in the zero-state. Similar behavior in PCI were observed for $A R L_{0}=200,500$, 1000 and $\delta_{\max }=2,3$.

Similarly, in Table 10 the ratios of the OOC ARLs (see Equation (15)) also indicate that the competing schemes listed in Table 1 yield larger OOC ARLs to a larger degree or extent compared to the MSS scheme. For instance, as $H$ varies from 1 to 20 , the DR scheme in zero-state is worse-off by a range of $83.30 \%$ to $142.81 \%$, however, in steady-state the DR (and WS) scheme is only worse- 
off by $15.58 \%$ to $31.94 \%$ i.e. in steady-state the competing schemes do not yield as much large

difference in the OOC ratios of the ARLs in comparison to the MSS (and AR) scheme.

Table 9: The zero-state and steady-state $P C I$ values of the 2-of- $(H+1)$ synthetic and runs-rules $\bar{X}$ charts for $\delta_{\max }=5$ when $H=1,2, \ldots, 20$ and $A R L_{0}=370.4$

\begin{tabular}{|c|c|c|c|c|c|c|c|c|c|c|c|c|}
\hline \multirow[b]{2}{*}{$H$} & \multicolumn{8}{|c|}{ Zero-state } & \multicolumn{4}{|c|}{ Steady-state } \\
\hline & DR & $\mathrm{KL}$ & $\mathrm{MC1}$ & $\mathrm{AR}$ & WS & DW & MC2 & MSS & DR \& WS & KL \& DW & MC1 \& MC2 & AR \& MSS \\
\hline 1 & 1.8286 & 1.6027 & 1.6027 & 1.6027 & 1.2010 & 1.0000 & 1.0000 & 1.0000 & 1.1377 & 1.0000 & 1.0000 & 1.0000 \\
\hline 2 & 1.9608 & 1.7424 & 1.7391 & 1.6960 & 1.2207 & 1.0365 & 1.0338 & 1.0000 & 1.1486 & 1.0255 & 1.0236 & 1.0000 \\
\hline 3 & 2.0371 & 1.8224 & 1.8170 & 1.7394 & 1.2356 & 1.0609 & 1.0568 & 1.0000 & 1.1590 & 1.0431 & 1.0402 & 1.0000 \\
\hline 4 & 2.0889 & 1.8764 & 1.8692 & 1.7637 & 1.2466 & 1.0783 & 1.0732 & 1.0000 & 1.1673 & 1.0563 & 1.0526 & 1.0000 \\
\hline 5 & 2.1262 & 1.9154 & 1.9069 & 1.7784 & 1.2543 & 1.0913 & 1.0854 & 1.0000 & 1.1738 & 1.0665 & 1.0622 & 1.0000 \\
\hline 6 & 2.1544 & 1.9448 & 1.9351 & 1.7875 & 1.2600 & 1.1013 & 1.0947 & 1.0000 & 1.1790 & 1.0745 & 1.0697 & 1.0000 \\
\hline 7 & 2.1765 & 1.9711 & 1.9572 & 1.7933 & 1.2643 & 1.1113 & 1.1021 & 1.0000 & 1.1831 & 1.0824 & 1.0758 & 1.0000 \\
\hline 8 & 2.1944 & 1.9868 & 1.9751 & 1.7968 & 1.2679 & 1.1157 & 1.1080 & 1.0000 & 1.1866 & 1.0867 & 1.0810 & 1.0000 \\
\hline 9 & 2.2094 & 2.0029 & 1.9902 & 1.7992 & 1.2709 & 1.1215 & 1.1134 & 1.0000 & 1.1896 & 1.0916 & 1.0855 & 1.0000 \\
\hline 10 & 2.2229 & 2.0169 & 2.0034 & 1.8007 & 1.2737 & 1.1266 & 1.1182 & 1.0000 & 1.1923 & 1.0959 & 1.0895 & 1.0000 \\
\hline 11 & 2.2346 & 2.0293 & 2.0151 & 1.8016 & 1.2763 & 1.1314 & 1.1226 & 1.0000 & 1.1946 & 1.0998 & 1.0931 & 1.0000 \\
\hline 12 & 2.2456 & 2.0406 & 2.0254 & 1.8021 & 1.2790 & 1.1359 & 1.1267 & 1.0000 & 1.1969 & 1.1035 & 1.0965 & 1.0000 \\
\hline 13 & 2.2556 & 2.0512 & 2.0353 & 1.8025 & 1.2817 & 1.1403 & 1.1308 & 1.0000 & 1.1990 & 1.1069 & 1.0996 & 1.0000 \\
\hline 14 & 2.2654 & 2.0612 & 2.0446 & 1.8028 & 1.2844 & 1.1446 & 1.1347 & 1.0000 & 1.2012 & 1.1101 & 1.1026 & 1.0000 \\
\hline 15 & 2.2745 & 2.0706 & 2.0533 & 1.8029 & 1.2874 & 1.1489 & 1.1387 & 1.0000 & 1.2031 & 1.1132 & 1.1054 & 1.0000 \\
\hline 16 & 2.2834 & 2.0797 & 2.0617 & 1.8031 & 1.2902 & 1.1530 & 1.1425 & 1.0000 & 1.2049 & 1.1161 & 1.1081 & 1.0000 \\
\hline 17 & 2.2917 & 2.0884 & 2.0697 & 1.8031 & 1.2932 & 1.1571 & 1.1464 & 1.0000 & 1.2068 & 1.1190 & 1.1108 & 1.0000 \\
\hline 18 & 2.2999 & 2.0967 & 2.0773 & 1.8032 & 1.2960 & 1.1612 & 1.1501 & 1.0000 & 1.2086 & 1.1217 & 1.1132 & 1.0000 \\
\hline 19 & 2.3080 & 2.1047 & 2.0846 & 1.8032 & 1.2990 & 1.1652 & 1.1540 & 1.0000 & 1.2103 & 1.1248 & 1.1157 & 1.0000 \\
\hline 20 & 2.3155 & 2.1126 & 2.0918 & 1.8032 & 1.3020 & 1.1691 & 1.1576 & 1.0000 & 1.2121 & 1.1269 & 1.1180 & 1.0000 \\
\hline
\end{tabular}

Table 10: The zero-state and steady-state $A R A R L$ values of the 2-of-(H+1) synthetic and runs-rules $\bar{X}$ charts for $\delta_{\max }=5$ when $H=1,2, \ldots, 20$ and $A R L_{0}=370.4$

\begin{tabular}{|c|c|c|c|c|c|c|c|c|c|c|c|c|}
\hline \multirow[b]{2}{*}{$H$} & \multicolumn{8}{|c|}{ Zero-state } & \multicolumn{4}{|c|}{ Steady-state } \\
\hline & DR & KL & $\mathrm{MC1}$ & AR & WS & DW & $\mathrm{MC2}$ & MSS & DR \& WS & KL \& DW & MC1 \& MC2 & AR \& MSS \\
\hline 1 & 1.8330 & 1.6257 & 1.6257 & 1.6257 & 1.1790 & 1.0000 & 1.0000 & 1.0000 & 1.1558 & 1.0000 & 1.0000 & 1.0000 \\
\hline 2 & 1.9729 & 1.7552 & 1.7518 & 1.7044 & 1.2145 & 1.0385 & 1.0359 & 1.0000 & 1.1845 & 1.0328 & 1.0304 & 1.0000 \\
\hline 4 & 2.1225 & 1.8908 & 1.8827 & 1.7562 & 1.2651 & 1.0902 & 1.0848 & 1.0000 & 1.2274 & 1.0783 & 1.0733 & 1.0000 \\
\hline 5 & 2.1696 & 1.9342 & 1.9245 & 1.7669 & 1.2812 & 1.1078 & 1.1014 & 1.0000 & 1.2421 & 1.0945 & 1.0885 & 1.0000 \\
\hline 6 & 2.2066 & 1.9687 & 1.9574 & 1.7735 & 1.2933 & 1.1219 & 1.1146 & 1.0000 & 1.2537 & 1.1074 & 1.1006 & 1.0000 \\
\hline 9 & 2.2811 & 2.0410 & 2.0259 & 1.7816 & 1.3162 & 1.1512 & 1.1421 & 1.0000 & 1.2763 & 1.1353 & 1.1264 & 1.0000 \\
\hline 10 & 2.2997 & 2.0592 & 2.0431 & 1.7827 & 1.3217 & 1.1588 & 1.1493 & 1.0000 & 1.2818 & 1.1423 & 1.1329 & 1.0000 \\
\hline 11 & 2.3160 & 2.0756 & 2.0586 & 1.7834 & 1.3267 & 1.1659 & 1.1559 & 1.0000 & 1.2865 & 1.1487 & 1.1388 & 1.0000 \\
\hline 12 & 2.3313 & 2.0907 & 2.0725 & 1.7836 & 1.3315 & 1.1725 & 1.1621 & 1.0000 & 1.2908 & 1.1547 & 1.1444 & 1.0000 \\
\hline 13 & 2.3453 & 2.1049 & 2.0858 & 1.7839 & 1.3364 & 1.1788 & 1.1681 & 1.0000 & 1.2950 & 1.1602 & 1.1495 & 1.0000 \\
\hline 14 & 2.3588 & 2.1183 & 2.0984 & 1.7841 & 1.3410 & 1.1851 & 1.1739 & 1.0000 & 1.2990 & 1.1654 & 1.1543 & 1.0000 \\
\hline 20 & 2.4281 & 2.1873 & 2.1621 & 1.7844 & 1.3689 & 1.2198 & 1.2068 & 1.0000 & 1.3194 & 1.1925 & 1.1792 & 1.0000 \\
\hline
\end{tabular}


Davis and Woodall [10] stated that “... as $H$ increases, a synthetic $\bar{X}$ chart behaves more and more like an $\bar{X}$ chart" - this might be the case with WS, DW and MC2 schemes. For the newly proposed MSS synthetic $\bar{X}$ chart, we see that this statement does not hold because as $H$ increases, the overall performance statistics decrease in an opposite direction as compared to the basic $\bar{X}$ chart and converge to some value, no matter how large $H$ is. However, the overall performance of the WS, DW and MC2 schemes tend to decrease, at first, and then at some point, increase towards the overall performance of the basic $\bar{X}$ chart - this phenomenon is summarized in Table 8 .

\section{Application example}

To illustrate the utility and the application of the proposed chart, we consider a well-known dataset from Montgomery [20] on the inside diameters of piston rings manufactured by a forging process. This data set contains twenty-five retrospective or Phase I samples, each of size five, that were collected when the process was thought to be IC. These data are considered to be the Phase I reference data for which a goodness of fit test for normality is not rejected. This data set also contains fifteen prospective (Phase II) samples each of five observations $(n=5)$. Firstly, $\quad$ though, when the distribution parameters of a particular process are unknown, it is generally accepted that there are two phases of application of control charts, namely Phase I and Phase II, see Chakraborti et al. [7] for further discussion on this. 


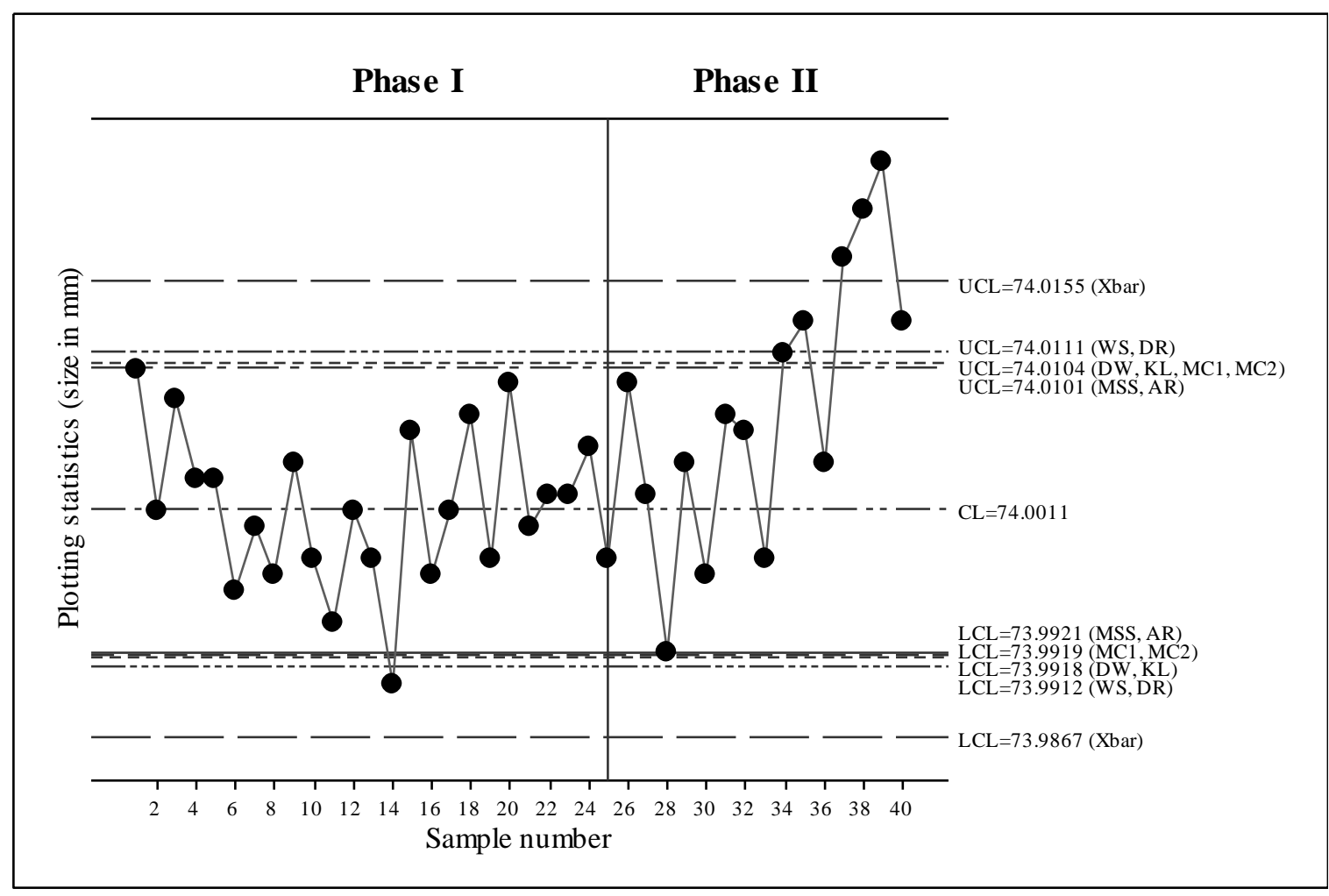

Figure 5: The synthetic and runs-rules $\bar{X}$ charts for the piston ring data

Here we use the piston rings data to illustrate the construction and operation of the MSS, MC2, DW, WS, AR, MC1, KL and DR schemes in steady-state mode, since Champ [8] stated that a more realistic mode of analysis is the steady-state. Note though, zero-state analysis follows in a similar fashion by using the chart design constants (i.e. $H$ and $k$ ) in Table 3 . Assume that the desired $A R L_{0}$ is 370.4 and we are interested in a $2-o f-3$ runs-type chart (i.e. $H=2$ ). The sample mean and sample standard deviation estimated from an IC Phase I data are 74.001 and 0.005 , respectively. Then, using the values of $k$ in Panel (b) of Table 3 for $H=2, k=3$ for the $\bar{X}$ chart and Equation (1), it follows that the control limits for each of these schemes are as given in Figure 5. From Figure 5, it is clear that the Phase I data are IC, as no OOC signal is detected by each of the 2-of-3 runs-type schemes or the 1 -of- 1 for the $\bar{X}$ chart, for the first 25 observations. However, in Phase II, the MSS, MC2, DW, AR, MC1 and KL schemes give an OOC signal at sample number 35, as two out of three consecutive plotting statistics are above the respective UCLs. Note though, the non-sidesensitive DR and WS schemes only signal later at sample number 37 - similar to the first OOC 
signal by the $\bar{X}$ chart. This supports the fact that the side-sensitive charts are more efficient than their non-side-sensitive counterparts.

\section{Conclusion}

The literature has witnessed a tremendous growth in synthetic charts in the last few years since many practitioners prefer waiting until the occurrence of a second nonconforming sample beyond the control limits before looking for an assignable cause. Hence, in this paper, we considered the performance of such schemes that take into account two nonconforming samples falling outside the control limits (i.e. the 2 - $o f-(H+1)$ runs-rules schemes for $H=1,2, \ldots, 20$ - with or without a head-start) before issuing an OOC signal. We then proposed a MSS synthetic $\bar{X}$ chart that issues a signal when all the consecutive $H+1$ samples that lead to an OOC event are on one side of the center line. Using the zero-state and steady-state $A R L, E Q L, P C I$ and $A R A R L$ as performance measures, we showed that this new synthetic $\bar{X}$ chart has either the same or better overall OOC performance than the other competing Shewhart-type $\bar{X}$ schemes that take into account two nonconforming samples falling outside the control limits before issuing an OOC signal. Note though, the performance of this new synthetic $\bar{X}$ chart is more pronounced in zero-state and relatively lower in steady-state, but it is uniformly better than the three classes of synthetic $\bar{X}$ charts currently available in the literature. Based on this study, the MSS synthetic $\bar{X}$ chart warrants being a strong contender to practical applications where 2-of- $(H+1)$ runs-rules $\bar{X}$ schemes with or without a head-start feature are currently used.

The results in this article are based on the assumptions of iid normally distributed observations; hence if the assumptions do not hold, the above results may have to be re-examined. A synthetic $\bar{X}$ chart with warning limits (in addition to control limits in Equation (1)) similar to the runs-rules scheme in Antzoulakos and Rakitzis [4] has been investigated and reported in Shongwe and Graham [23]. For future research purposes, we will investigate the double sampling MSS chart and compare its performance to the double sampling schemes in Khoo et al. [15] (for WS scheme) 
and Costa and Machado [9] (for MC2 scheme). In addition, the effect of parameter estimation may be conducted for both fixed and double sampling schemes, similar to that done in Zhang et al. [27] and You et al. [26], respectively.

\section{Acknowledgements}

The authors are indebted to the reviewer and the Editors who carefully read the earlier draft and found some mistakes. Part of this work was supported by the SARCHI Chair at the University of Pretoria. Sandile Shongwe's research was supported in part by the National Research Foundation and Department of Science and Technology's Innovation Doctoral scholarship (SFH14081591713) and Marien Graham's research was supported in part by the National Research Foundation (Thuthuka programme: TTK20100724000013247, Grant number: 76219).

\section{References}

1. Abbas, N., Riaz, M. and Does, R.J.M.M. (2011). Enhancing the performance of EWMA charts. Quality and Reliability Engineering International, 27 (6), 821-833.

2. Abbas, N., Riaz, M., Does, R.J.M.M. (2015). Enhancing the performance of exponentially weighted moving average charts: Discussion. Quality and Reliability Engineering International, $31(4), 721-722$.

3. Abujiya, M.R., Farouk, A.U., Lee, M.H. and Mohamad, I. (2013). On the sensitivity of Poisson EWMA control chart. International Journal of Humanities and Management Sciences, 1 (1), $18-22$.

4. Antzoulakos, D.L. and Rakitzis, A.C. (2008). The revised m-of-k runs rule. Quality Engineering, 20 (1), 75-81.

5. Antzoulakos, D.L. and Rakitzis, A.C. (2008). The modified $r$ out of $m$ control chart. Communications in Statistics - Simulation and Computation, 37 (2), 396-408.

6. Bourke, P.D. (1991). Detecting a shift in fraction nonconforming using run-length control charts with 100\% inspection. Journal of Quality Technology, 23 (3), 225-238.

7. Chakraborti, S., Human, S.W. and Graham, M.A. (2009). Phase I statistical process control charts: An overview and some results. Quality Engineering, 21 (1), 52-62.

8. Champ, C.W. (1992). Steady-state run length analysis of a Shewhart quality control chart with supplementary runs rules. Communications in Statistics - Theory and Methods, 21 (3), 765-777. 
9. Costa, A.F.B. and Machado, M.A.G. (2015). The steady-state behavior of the synthetic and sidesensitive double sampling $\bar{X}$ charts. Quality and Reliability Engineering International, 31 (2), 297-303.

10. Davis, R.B. and Woodall, W.H. (2002). Evaluating and improving the synthetic control chart. Journal of Quality Technology, 34 (2), 200-208.

11. Derman, C. and Ross, S.M. (1997). Statistical Aspects of Quality Control. Academic Press, San Diego, CA.

12. Fu, J.C. and Lou W.Y.W. (2003). Distribution Theory of Runs and Patterns and Its Applications: A Finite Markov Chain Imbedding Approach. Singapore: World Scientific Publishing.

13. Haq, A., Brown, J. and Moltchanova, E. (2015). New synthetic EWMA and synthetic CUSUM control charts for monitoring the process mean. Quality and Reliability Engineering International, DOI: 10.1002/qre.1747.

14. Khoo, M.B.C., Castagliola, P., Liew, J.Y., Teoh, W.L. and Maravelakis P.E. (2015). A study on EWMA charts with runs-rules - the Markov chain approach. Communications in Statistics Theory and Methods; Accepted.

15. Khoo, M.B.C., Lee, H.C., Wu, Z., Chen, C-.H. and Castagliola, P. (2011). A synthetic double sampling control chart for the process mean. IIE Transactions, 43 (1), 23-38.

16. Klein, M. (2000). Two alternatives to the Shewhart $\bar{X}$ control chart. Journal of Quality Technology, 32 (4), 427-431.

17. Low, C.K., Khoo, M.B.C., Teoh, W.L. and Wu, Z. (2012). The revised m-of-k runs-rule based on median run length. Communications in Statistics - Simulation and Computation, 41 (8): 1463-1477.

18. Machado, M.A.G. and Costa A.F.B. (2014). Some comments regarding the synthetic chart. Communications in Statistics - Theory and Methods, 43 (14), 2897-2906.

19. Machado, M.A.G. and Costa A.F.B. (2014). A side-sensitive synthetic chart combined with an $\bar{X}$ chart. International Journal of Production Research, 52 (11), 3404-3416.

20. Montgomery, D.C. (2013). Statistical Quality Control: A Modern Introduction (7th edn). John Wiley \& Sons, Singapore Pte. Ltd.

21. Riaz, M., Abbas, N. and Does, R.J.M.M. (2011) Improving the performance of CUSUM charts. Quality and Reliability Engineering International, 27 (4), 415-424.

22. Scariano, S.M. and Calzada, M.E. (2009). The generalized synthetic chart. Sequential Analysis, 28 (1), 54-68. 
23. Shongwe, S.C. and Graham, M.A. (2015). On the performance of Shewhart-type synthetic and runs-rules charts combined with an $\bar{X}$ chart. Quality and Reliability Engineering International, DOI: 10.1002/qre.1836; Accepted.

24. Wu, Z. and Spedding, T.A. (2000). A synthetic control chart for detecting small shifts in the process mean. Journal of Quality Technology, 32 (1), 32-38.

25. Wu, Z., Yang, W., Jiang, W. and Khoo M.B.C. (2008). Optimization designs of the combined Shewhart-CUSUM control charts. Computational Statistics \& Data Analysis, 53 (2), 496-506.

26. You, H.W., Khoo, M.B.C., Lee, M.H. and Castagliola, P. (2015). Synthetic double sampling $\bar{X}$ chart with estimated parameters. Quality Technology \& Quantitative Management, Accepted.

27.Zhang, Y., Castagliola, P., Wu, Z. and Khoo, M.B.C. (2011). The synthetic $\bar{X}$ chart with estimated parameters. IIE Transactions, 43 (9), 676-687. 